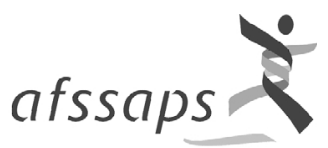

\title{
Transfusion de globules rouges homologues : produits, indications, alternatives
}

\section{Recommandations}

\section{GROUPE DE TRAVAIL}

Pr LIENHART André, Président, Anesthésiste-Réanimateur, Paris Dr NOUYRIGAT Emmanuel, Responsable de Projet, Afssaps Dr DUMARCET Nathalie, Responsable de Projet, Afssaps Dr ANDREU Georges, Hémobiologiste, Tours Pr BARON Jean-François, Anesthésiste-Réanimateur, Paris Pr BENHAMOU Dan, Anesthésiste-Réanimateur, Le Kremlin-Bicêtre Dr COURTOIS Françoise, Hémobiologiste, Paris

Dr DE MONTALEMBERT-RIDET Mariane, Hémobiologiste, Paris Pr GALACTEROS Frédéric, Génétique Médicale, Créteil Dr GUYARD Marie-Françoise, Anesthésiste-Réanimateur, Rouen Pr IFRAH Norbert, Hématologue, Angers

Dr LEJUS Corinne, Anesthésiste-Réanimateur Pr OZIER Yves, Anesthésiste-Réanimateur, Paris Pr SIMEONI Umberto, Néonatalogiste, Marseille Dr SPAETH Dominique, Oncologue, Vandoeuvre-les-Nancy Pr VALLET Benoît, Anesthésiste-Réanimateur, Lille Dr DAVID Bernard, Afssaps

Dr DENIS Catherine, Afssaps Dr OUNNOUGHENE Nadra, Afssaps

Dr SANDID Imad, Afssaps

Dr SAURA Christine, Afssaps

\section{GROUPE DE LECTURE}

Dr BLANLOEIL Yvonnick, Anesthésiste-Réanimateur, Nantes Dr BONNET Vincent, Anesthésiste-Réanimateur, Paris Dr CARTRON Guillaume, Oncologue, Saint-Avertin Dr CHABANEL Anne, Docteur ès Sciences, Paris Dr CHENAIS Françoise, Hémobiologiste, Grenoble Pr CONSEILLER Christian, Anesthésiste-Réanimateur, Paris Dr DRISS Françoise, Hémobiologiste, Le Kremlin-Bicêtre Dr DROUET Nicolas, Anesthésiste-Réanimateur, Meylan Dr DUCLOY-BOUTHORS Anne-Sophie, Anesthésiste-Réanimateur, Lille

Dr DUMEIX Jean-Marc, Anesthésiste-Réanimateur, Saint-Rémy Dr GENTILI Marc, Anesthésiste-Réanimateur, Saint-Grégoire Pr HARDY Jean-François, Anesthésiste-Réanimateur, Montréal Pr JOUSSEMET Marcel, Hémobiologiste, Clamart Pr KESSLER Michèle, Néphrologue, Vandoeuvre-les-Nancy Dr LE ROLLE Thérèse, Anesthésiste-Réanimateur, Angers Pr LEPORRIER Michel, Hématologue, Caen
Pr MARTIN Claude, Anesthésiste-Réanimateur, Marseille Pr MILPIED Noel, Hématologue, Nantes Pr MORIETTE Guy, Néonatalogiste, Paris Dr PALOT Maryse, Anesthésiste-Réanimateur, Reims Dr QUARANTA Jean-François, Hémobiologiste, Nice Dr RIALLAND Xavier, Pédiatre, Angers Pr ROSE Christian, Hématologue, Lille Pr ROUGER Philippe, Immunologiste, Paris Dr THURET Isabelle, Pédiatre, Marseille Dr VAN DER LINDEN Philippe, Anesthésiste-Réanimateur, Charleroi

Dr VIRY-BABEL Françoise, Hémobiologiste, Nancy

Pr WAUTIER Jean-Luc, Hématologue, Paris

\section{COMITE DE VALIDATION}

Pr BOUVENOT Gilles, Président, Thérapeutique, Marseille Pr BERGMANN Jean-François, Vice-Président, Thérapeutique, Paris

Pr CAULIN Charles, Président de la Commission d'AMM, Paris

Pr DUPUIS Bernard, Président de la Commission de Transparence, Lille

Pr AUBIER Michel, Pneumologue, Paris

Pr BANNWARTH Bernard, Pharmacologue, Rhumatologue, Bordeaux

Dr CAMELLI Bruno, Généraliste, Paris

Pr FUNCK-BRENTANO Christian, Pharmacologue Clinicien, Cardiologue, Paris

Pr LE JEUNNE Claire, Thérapeutique, Paris

Pr PETIT Michel, Psychiatre, Sotteville-lès-Rouen

Dr REVEILLAUD Olivier, Généraliste, Bièvres

Pr RICHÉ Christian, Pharmacologue, Brest

Pr THERY Claude, Cardiologue, Lille

Dr TREMOLIERES François, Infectiologue, Interniste, Mantes-laJolie

Dr WONG Olivier, Généraliste, Paris

Dr BELORGEY Chantal, Afssaps

Dr DENIS Catherine, Afssaps

Dr DUMARCET Nathalie, Afssaps

Dr NOUYRIGAT Emmanuel, Afssaps

Dr ROSTOKER Guy, Afssaps

Dr TROUVIN Jean-Hugues, Afssaps 


\section{Liste des abréviations}

AHA : Anémie Hémolytique Acquise

AMM : Autorisation de Mise sur le Marché

AVC : Accident Vasculaire Cérébral

$\mathrm{CaO}_{2}$ : Contenu du Sang Artériel en Oxygène

$\mathrm{CO}_{2}$ : Dioxyde de Carbone

CIVD : Coagulation Intravasculaire Disséminée

CGR : Concentré de Globules Rouges

CMV : Cytomégalovirus

DS : Déviation Standard

EPO : Erythropoïétine

GR : Globules Rouges

GVH : Graft Versus Host Disease (= maladie du greffon contre l'hôte)

[Hb] : Concentration en Hémoglobine

Ht : Hématocrite

HTLV : Human T-cell lymphoma virus (= Virus des Lymphocytes $\mathrm{T}$ humains)
OAP : Edème Aigu du Poumon

$\mathrm{O}_{2}$ : Oxygène

$\mathrm{PaO}_{2}$ : Pression partielle de l' $\mathrm{O}_{2}$ du sang artériel

PFC : Plasma Frais Congelé

PSL : Produits Sanguins Labiles

RAI : Recherche d'Agglutinines Irrégulières

RH : Rhésus

SAGM : Milieu de Conservation Saline, Adénine, Glucose, Mannitol

$\mathrm{SaO}_{2}$ : Saturation en Oxygène du Sang Artériel

$\mathrm{S}_{\bar{v}} \mathrm{O}_{2}$ : Saturation en Oxygène du Sang Veineux Mêlé

TAP : Transfusion Autologue Programmée

$\mathrm{TaO}_{2}$ : Transport Artériel de l'Oxygène aux Tissus

UA : Unité Adulte

UE : Unité Enfant

$\mathrm{VO}_{2}$ : Consommation d'Oxygène

VST : Volume Sanguin Total

\section{Méthodologie}

L'ordonnance $\mathrm{n}^{\circ}$ 96-345 du 24 avril 1996 relative à la maîtrise médicalisée des dépenses de soins a confié à l'Agence Française de Sécurité Sanitaire des Produits de Santé (Afssaps) la mission d'établir les recommandations de bonne pratique et les références médicales, concernant le médicament et les produits biologiques. Elle stipule d'autre part que les recommandations de bonne pratique et références existantes doivent être régulièrement actualisées, en fonction des données nouvelles de la science.

C'est dans ce contexte que l'Afssaps propose une actualisation des recommandations de bonne pratique et références : «Indications et contre-indications des transfusions de produits sanguins labiles », établies par l'Agence Nationale d'Accréditation et d'Evaluation en Santé (ANAES) et l'Agence Française du Sang (AFS) en 1997, et notamment sur la transfusion de globules rouges.

Ces recommandations définissent une stratégie médicale optimale en fonction de l'état actuel des connaissances et précisent ce qui est utile ou inutile, voire dangereux, de faire dans une situation clinique donnée.

Ces recommandations résultent de l'analyse des données actuelles de la science issues de la littérature, et prennent en compte les évaluations réalisées pour délivrer l'autorisation de mise sur le marché (AMM) des médicaments concernés, apprécier le service médical rendu (SMR) et élaborer les fiches de transparence. Les sociétés savantes ont été consultées (Société Française d'Anesthésie-Réanimation, Société de Réanimation de Langue Française, Société Française d'Hématologie, Société Française d'Hémaphérèse, Collège Français des Hématologistes, Société Française d'Hématologie et d'Immuno-Hématologie Pédiatrique, Société Française de Pédiatrie, Fédération Nationale des Pédiatres Néonatalogistes, Société Française du Cancer, Société Française de Néphrologie, Société Française de Transfusion Sanguine, Société Française de Vigilance des Thérapeutiques de Transfusion, Association Développement Transfusion Sanguine), ainsi que l'Etablissement Français du Sang pour proposer des représentants susceptibles de participer aux groupes.

Le groupe de travail constitué par l'Afssaps a regroupé des experts de compétence (anesthésie-réanimation, hémobiologie, hématologie, néonatalogie, oncologie), de mode d'exercice (hospitalo-universitaires ou hospitaliers) et d'origine géographique divers, ainsi que des représentants de l'Afssaps. Les experts ont analysé la littérature et rédigé le document sous la direction d'un président de groupe et l'encadrement d'un responsable de projet.

La recherche bibliographique a été réalisée par interrogation systématique des banques de données Medline. Elle a identifié préférentiellement les recommandations thérapeutiques, les conférences de consensus, les essais cliniques, les méta-analyses, 
les analyses de décision et les revues de synthèse, publiés en langue française ou anglaise après 1997.

De plus, les listes de références citées dans les articles déjà identifiés ont été consultées et les membres du groupe de travail et du groupe de lecture ont pu transmettre d'autres articles.

La recherche bibliographique automatisée était basée sur les mots clés suivants :

erythrocyte transfusion/erythrocytes/leucoreduction, leucocytes reduction/cytomegalovirus/cancer/immune function /irradiation/anaphylactic/graft-versus-host reaction disease/anaemia/blood transfusion/blood component transfusion/blood transfusion, autologous/blood transfusion, intrauterine/acute disease/'acute blood loss'/chronic disease/acute, severe anaemia/haemodilution/haemoglobin/haematocrit/tolerance, complications, respiratory distress/myocardial ischaemia/fatigue, rehabilitation/cerebral stroke/hyperkalaemia/children, infants/ $\mathrm{HemoCue}^{\circledR}$, haemoglobimeter/haemorrhage/anaesthesia/emergencies/intraoperative care/intraoperative period/intensive care/ intensive care units/resuscitation/blood transfusion, utilisation/ emergency medical services/patient care/neoplasms, therapy/ haematological diseases, therapy/anaemia, neonatal/neonatology/infant, newborn/intensive care, neonatal/intensive care units, neonatal/‘neonatal diseases and abnormalities'/erythropoietin.

Au total, 353 références supplémentaires ont été utilisées pour l'élaboration du texte par rapport à celui de l'ANAES de 1997.
L'argumentaire et les recommandations de ce travail ont été établis par le groupe selon la méthodologie proposée par l'ANAES (ANAES : Les recommandations pour la pratique clinique - Base méthodologique pour leur réalisation en France - 1999 ; Guide d'analyse de la littérature et gradations des recommandations - 2000). Chaque article a été analysé en appréciant la qualité méthodologique des études, afin d'affecter à chacun un niveau de preuve scientifique. Pour ce faire des grilles de lecture destinées à apprécier la qualité méthodologique et le niveau de preuve scientifique des documents ont été utilisées.

Les grades $\mathrm{A}, \mathrm{B}$, et $\mathrm{C}$ sont attribués aux recommandations selon le niveau de preuve scientifique attribué aux études sur lesquelles elles reposent (tableau I). Lorsque les données de la littérature sont insuffisantes ou incomplètes, les recommandations sont basées sur un Accord professionnel pour prendre en compte l'état des pratiques et les opinions d'experts.

Le texte a été soumis à un groupe de lecture avant d'être finalisé. Le groupe de lecture est composé d'experts de compétence, de mode d'exercice et d'origine géographique divers. Les experts de ce groupe de lecture, consultés par courrier, ont apprécié la qualité méthodologique et la validité scientifique du contenu, ainsi que la lisibilité, la faisabilité et l'applicabilité du texte. Leurs remarques ont été transmises à l'ensemble du groupe de travail qui a pu modifier son texte et validé le document final.

Le texte a ensuite été soumis à l'avis du Comité de Validation des Recommandations et Références Médicales de l'Afssaps.

Tableau I.

\begin{tabular}{|c|c|}
\hline Niveau de preuve scientifique des études & Force des recommandations (grade) \\
\hline Niveau 1 & A : Preuve scientifique établie \\
\hline \multicolumn{2}{|l|}{ Essais comparatifs randomisés de forte puissance } \\
\hline \multicolumn{2}{|l|}{ Méta-analyse d'essais comparatifs randomisés } \\
\hline \multicolumn{2}{|l|}{ Analyse de décision basée sur des études bien menées } \\
\hline Niveau 2 & B : Présomption scientifique \\
\hline \multicolumn{2}{|l|}{ Essais comparatifs randomisés de faible puissance } \\
\hline \multicolumn{2}{|l|}{ Etudes comparatives non randomisées bien menées } \\
\hline \multicolumn{2}{|l|}{ Etudes de cohorte } \\
\hline \multicolumn{2}{|l|}{ Niveau 3} \\
\hline \multicolumn{2}{|l|}{ Etudes cas-témoin } \\
\hline Niveau 4 & C : Faible niveau de preuve scientifique \\
\hline \multicolumn{2}{|l|}{ Etudes comparatives comportant des biais importants } \\
\hline \multicolumn{2}{|l|}{ Etudes rétrospectives } \\
\hline \multicolumn{2}{|l|}{ Séries de cas } \\
\hline Etudes épidémiologiques descriptives (transversale, longitudinale) & \\
\hline
\end{tabular}




\section{Introduction}

Comme nombre d'actes médicaux, l'acte de transfusion de globules rouges comporte des risques, tout comme l'abstention de le réaliser. Il n'en demeure pas moins que de telles décisions doivent être prises, et c'est le devoir du médecin que de hiérarchiser les différents risques en fonction de la situation du patient et de l'état actuel des connaissances médicales, ceci afin de faire bénéficier la personne soignée du meilleur rapport bénéfice/risque de la thérapeutique proposée.

Ainsi, l'Afssaps s'est posée la question du risque de transmission d'agents non viraux, tel le nouveau variant de la maladie de Creutzfeldt-Jakob. Face à un risque qui n'est que potentiel à ce jour, et en tous cas largement inconnu, deux attitudes transfusionnelles sont également contestables : la première, «trop large », consisterait à attendre que le risque de transmission se réalise, avant de tenter de le réduire ; la deuxième, « trop restrictive », augmenterait en contrepartie d'autres risques bien connus. Ces différents écueils à éviter expliquent que, entre autres mesures, l'Afssaps a souhaité faire le point des acquisitions nouvelles en matière de transfusion des globules rouges et de ses alternatives, comme la transfusion de sang autologue, l'érythropoiétine et le traitement martial.

Ces recommandations ont pour objectif d'améliorer la qualité de la transfusion des globules rouges en aidant le praticien dans sa prise de décision et en contribuant à une homogénéisation des pratiques.

\section{Les différents produits érythrocytaires disponibles et leurs indications}

PSL érythrocytaires :

- obtenus soit à partir d'une unité de sang total, soit à l'aide d'un séparateur de cellules ;

- contiennent toujours un anticoagulant, en général le CPD (citrate, phosphate, dextrose) ;

- ne font pas actuellement l'objet d'inactivation virale.

Chez les patients recevant plusieurs types de PSL, il convient d'assurer systématiquement la cohérence des indications des différentes transformations et qualifications pour chaque produit prescrit (tableau II).

Les principales caractéristiques des produits érythrocytaires transformés sont détaillées en Annexe I.

\subsection{Transformations applicables aux produits érythrocytaires}

\subsubsection{Déleucocytation}

Obligation réglementaire en France depuis le $1^{\text {er }}$ avril 1998.
Tableau II. Liste des transformations et qualifications applicables aux produits érythrocytaires

\begin{tabular}{ll}
\hline Transformations & Qualifications \\
\hline Addition d'une solution supplémentaire de & Phénotypé \\
conservation en phase liquide & Compatibilisé \\
Déleucocytation & CMV négatif \\
Déplasmatisation & \\
Cryoconservation & \\
Irradiation par les rayonnements ionisants & \\
Préparation pédiatrique & \\
Réduction de volume & \\
Sang total reconstitué & \\
\hline CMV = cytomégalovirus.
\end{tabular}

CGR déleucocyté : suspension de GR obtenue aseptiquement par un processus associant au moins une étape de centrifugation (pour éliminer la majeure partie du plasma) et une étape de filtration (pour réaliser la déleucocytation). Il peut être réalisé à partir d'une unité de sang total ou d'un CGR après centrifugation. La solution de conservation est le SAGM. La solution de conservation et la déleucocytation permettent de diminuer les lésions de stockage des GR et d'allonger ainsi la durée de conservation des CGR à 42 jours. Le CGR-SAGM contient une quantité résiduelle de plasma (jusqu'à $25 \mathrm{~mL}$ ), des plaquettes (quantité résiduelle non standardisée) et des leucocytes $\left(\leq 1.10^{6}\right)$.

La déleucocytation est destinée à réduire de nombreux effets indésirables de la transfusion, en matière d'allo-immunisation anti-HLA, de réactions frissons-hyperthermie et de transmission transfusionnelle des virus intraleucocytaires (CMV, HTLV).

Bien que la transfusion de PSL possède un effet immunosuppresseur, la déleucocytation ne semble pas avoir d'effet pour la prévention des récidives de tumeur et son effet est controversé pour les infections post-opératoires.

\section{Indication des CGR déleucocytés}

Utilisés en l'absence d'indication de transformation ou de qualification particulière.

NB : Les CGR non déleucocytés sont autorisés pour certains protocoles de transfusion préalable à une greffe d'organe (arrêté du 30 mars 1998).

Chez le nouveau-né, la quantité de SAGM injectée est généralement inférieure au seuil de toxicité de ses divers composants ; il peut donc être largement utilisé, comme chez l'adulte.

Le CGR-SAGM déleucocyté n'est pas utilisé en cas de transfusion massive ( $\geq 1$ masse sanguine) (Accord professionnel). Dans ces situations, on peut avoir recours soit au sang total déleucocyté, soit au sang total reconstitué. 


\subsubsection{Déplasmatisation}

Les CGR déleucocytés déplasmatisés contiennent moins de $0,5 \mathrm{~g}$ de protéines extracellullaires, ont un contenu très réduit en plaquettes, sont périmés au bout de 6 heures.

Indications des CGR déleucocytés déplasmatisés (Accord professionnel)

- Patients intolérants aux protéines plasmatiques : antécédents de réactions transfusionnelles anaphylactiques majeures (urticaire étendu, bronchospasme et œdème de Quincke, choc anaphylactique, anticorps anti-IgA).

- Antécédents de purpura post-transfusionnel (la déplasmatisation assure une déplaquettisation).

Leur utilisation est discutée en cas d'antécédents de réactions transfusionnelles anaphylactiques mineures (urticaire, rash cutané) répétées. Elle n'est plus justifiée chez les patients atteints d'hémoglobinurie paroxystique nocturne.

Chez le nouveau-né, la déplasmatisation s'impose lorsque le produit à transfuser contient un anticorps potentiellement dangereux et s'il n'y a pas d'alternative.

Elle rend impossible la réalisation de "préparations pédiatriques » pour des raisons techniques; ses avantages doivent être mis en balance avec ceux de ces préparations (recours au donneur unique).

En raison de la présence d'anticorps maternels IgG dans le sang du fœtus et jusqu'à 3 mois après la naissance, les GR doivent être compatibles avec le groupe ABO de l'enfant et celui de la mère. Cette situation peut conduire, en particulier en cas de transfusion massive (exsanguino-transfusion), à utiliser la déplasmatisation pour réduire la concentration d'anticorps anti-A et/ou anti-B du CGR.

\subsubsection{Cryoconservation}

Elle permet la conservation à long-terme de GR viables et fonctionnels.

Selon la température de conservation $\left(-30^{\circ} \mathrm{C},-80^{\circ} \mathrm{C}\right.$ ou $\left.-130^{\circ} \mathrm{C}\right)$, la durée de stockage est de 4 mois à plus de 20 ans.

Il est possible de les conserver pendant 7 jours après décongélation à deux conditions :

- que les processus de congélation et de décongélation aient été réalisés dans des conditions de circuit fonctionnellement clos ;

- qu'une solution spécifique de conservation soit utilisée.

Les CGR ont une concentration de protéines plasmatiques analogue à celle des CGR déplasmatisés ( $<0,5 \mathrm{~g}$ par produit), et ont un faible taux résiduel de plaquettes et de leucocytes $\left(<10^{6}\right)$. Indications des CGR déleucocytés cryoconservés

- Patients de phénotype érythrocytaire rare ou exceptionnel («public négatif »)

- Patients ayant de multiples anticorps anti-érythrocytes.
Une réserve individuelle est constituée lorsqu'un traitement transfusionnel est prévisible (intervention programmée, grossesse débutante...).

Les CGR déleucocytés cryoconservés peuvent être employés dans les mêmes indications que les CGR déleucocytés déplasmatisés, mais leur emploi n'est pas opérationnel à grande échelle.

Il n'y a pas d'indication spécifique chez le nouveau-né.

\subsubsection{Irradiation par les rayonnements ionisants}

C'est une exposition de CGR déleucocytés à une dose de rayonnements ionisants de 25 à $45 \mathrm{~Gy}$ (réglementation française), afin de prévenir la survenue d'une maladie du greffon contre l'hôte $(\mathrm{GVH})$ post-transfusionnelle.

Indications des CGR déleucocytés irradiés (grade C)

- Patients porteurs d'un déficit immunitaire congénital cellulaire.

- Avant ou pendant un prélèvement de cellules souches hématopoḯtiques autologues, médullaires ou sanguines.

- Patients traités par greffe de cellules souches hématopoiétiques autologues ou allogéniques, dès le début du conditionnement, pendant au moins 1 an après autogreffe et à vie après allogreffe.

- Transfusion de CGR issus d'un don dirigé intrafamilial, quel que soit le degré de parenté entre donneur et receveur (obligation réglementaire).

En revanche, les indications suivantes ne font pas l'objet d'un Accord professionnel :

- maladie de Hodgkin en cours de traitement

- chimiothérapies pour lymphomes non hodgkiniens, leucémies aiguës ou tumeurs solides ;

- receveurs de greffe d'organe.

Dans ces deux dernières situations, elle ne paraît justifiée qu'en cas d'immunosuppression profonde.

Chez le foetus et le nouveau-né, l'irradiation est recommandée, en cas de transfusion intra-utérine, d'exsanguino-transfusion ou de transfusion massive ( $>1$ masse sanguine) chez le prématuré.

En onco-hématologie pédiatrique, les CGR sont habituellement irradiés de principe (Accord professionnel).

\subsubsection{Préparation pédiatrique}

C'est la division aseptique d'un CGR déleucocyté en plusieurs unités. Un seul don conduit à la production de plusieurs unités utilisables successivement pour un même patient.

Indication des préparations pédiatriques

- Transfusions répétées de GR chez le nouveau-né, afin de diminuer le nombre des donneurs. 


\subsubsection{Réduction de volume}

C'est l'élimination aseptique d'une partie du milieu de suspension d'un CGR déleucocyté par centrifugation. L'hématocrite (Ht) est alors compris entre 70 et $85 \%$.

\section{Indication à la réduction de volume}

Essentiellement le nouveau-né, dans deux circonstances :

- lorsque le contrôle du volume injecté doit être rigoureusement respecté ;

- transfusion massive, lorsqu'on désire éliminer la majeure partie de la solution de conservation en phase liquide.

\subsubsection{Sang total reconstitué}

C'est le mélange aseptique d'un CGR déleucocyté, soit avec de l'albumine à $4 \%$, soit avec du PFC. La reconstitution se fait habituellement avec du PFC pour prévenir les troubles de la coagulation que pourrait induire l'utilisation de l'albumine. Il n'existe pas d'étude montrant les bénéfices et les inconvénients de chacun des deux produits. Il doit être réalisé par l'établissement de transfusion sanguine (ETS).

Indication du sang total reconstitué

- Essentiellement le nouveau-né, pour la réalisation d'exsanguino-transfusions ou de techniques d'assistance cardiorespiratoire.

Rappel de la seule indication du «sang total $»$ : transfusion massive chez le nouveau-né, à condition que les groupes ABO du nouveau-né et de la mère permettent l'utilisation de GR et de plasma du même groupe.

\subsection{Qualifications applicables aux produits érythrocytaires}

\subsubsection{Phénotypage ${ }^{1}$}

Il s'applique à tous les CGR antigéno-compatibles avec le receveur pour les cinq antigènes : RH2 (C), RH3 (E), RH4 (c), RH5 (e) du système RH (Rh) et KEL1 (K) du système KELL (Kell).

Il est dit «étendu » lorsque, en plus du phénotype RH-KELL, au moins un antigène d'autres systèmes (Duffy, Kidd, MNS, Lewis, etc.) est antigéno-compatible avec le receveur.

Indications des CGR déleucocytés phénotypés RH et KELL Les CGR phénotypés RH et KELL sont :

- formellement indiqués chez :

- les patients ayant ou ayant eu un (des) allo-anticorps antiérythrocytaire(s) à l'exclusion de ceux jugés sans intérêt transfusionnel, afin de prévenir les accidents hémolytiques transfusionnels (obligation réglementaire) ;
- les femmes, de la naissance jusqu'à la fin de la période procréatrice, afin de prévenir l'apparition d'allo-anticorps antiérythrocytaires qui représentent un risque supplémentaire d'accident hémolytique foto-maternel (obligation réglementaire) ;

- les nouveau-nés, en présence d'un anticorps antiérythrocytaire (provenant de la mère), quel que soit le sexe.

- recommandés chez les patients recevant des transfusions itératives de CGR, afin de prévenir l'apparition d'allo-anticorps anti-érythrocytaires (grade $C$ ) ;

- $\quad$ souhaitables pour tout patient, quel que soit le sexe, ayant une espérance de vie raisonnable (Accord professionnel).

Non-indication des CGR phénotypés RH et KELL

- Patients dont la recherche de RAI est négative et dont l'espérance de vie est réduite.

Le respect du phénotype étendu à un ou plusieurs antigènes de groupe sanguin autre qu'ABO, RH et KELL est fonction des anticorps détectés chez les patients immunisés. Chez ces patients, il est souhaitable de respecter les phénotypes RH et KELL à titre préventif.

Le phénotype étendu aux antigènes FY1 $\left(\mathrm{Fy}^{\mathrm{a}}\right), \mathrm{JK} 1\left(\mathrm{Jk}^{\mathrm{a}}\right)$ et MNS3 (S), peut éventuellement être proposé à titre préventif chez les patients dépendant à long terme de transfusions de CGR, en particulier les patients drépanocytaires et les thalassémiques.

\subsubsection{Compatibilité}

L'épreuve directe de compatibilité de CGR phénotypés RH-KELL est une analyse non systématique complémentaire de la recherche des anticorps irréguliers anti-érythrocytaires. Elle consiste à tester le sérum du receveur vis-à-vis des GR contenus dans la tubulure du CGR à transfuser, puis d'attribuer à ce CGR la qualification «compatibilisé » si l'épreuve est négative. Le délai maximal de validité de cette épreuve est de 3 jours à partir de la date du prélèvement du receveur. Le CGR compatibilisé porte les mentions suivantes : identité du receveur, date de réalisation et durée de validité de l'épreuve (obligation réglementaire).

Indications des CGR déleucocytés compatibilisés

- Tout patient présentant ou ayant présenté ou suspecté de présenter un ou plusieurs allo-anticorps anti-érythrocytaire(s) vis-à-vis de CGR phénotypés RH-KELL, en dehors de l'urgence vitale (obligation réglementaire).

- Chez le nouveau-né, en présence d'anticorps anti-érythrocytaires, les CGR sont compatibilisés vis-à-vis du sérum de la mère, d'où proviennent les anticorps. En cas d'indisponibilité de ce sérum, celui de l'enfant est utilisé.

1 La nouvelle nomenclature des antigènes de groupes sanguins est utilisée dans ce texte ; l'ancienne nomenclature est mise entre parenthèses pour des raisons didactiques. La correspondance entre les deux nomenclatures se trouve en Annexe II. 


\subsubsection{CMV négatif}

Cette qualification s'applique aux CGR provenant de donneurs chez lesquels la recherche d'anticorps anti-CMV est négative au moment du don.

Sa disponibilité est limitée du fait de la séroprévalence élevée (50 à $80 \%$ ) des anticorps anti-CMV dans la population des donneurs de sang.

Il est à noter que le CMV ayant une localisation quasi-exclusive intra-leucocytaire (monocytes), la seule déleucocytation contribue à la prévention de sa transmission, sans que l'on puisse déterminer si l'association des deux méthodes apporte, ou non, une meilleure prévention.

\section{Indications}

En raison de la faible disponibilité du CGR CMV négatif et des doutes quant à sa supériorité sur le CGR déleucocyté, il apparaît nécessaire de hiérarchiser les indications.

L'indication suivante mérite d'être privilégiée (Accord professionnel) :

- allogreffes de cellules souches hématopoïétiques en situation où donneur et receveur sont CMV négatifs.

Dans la limite des produits disponibles (grade $C)$ :

- femmes enceintes CMV négatives ;

- prématurés de moins de 32 semaines d'âge gestationnel, lorsque la mère est séronégative pour le $\mathrm{CMV}$;
- receveurs de greffe de poumon, quel que soit leur statut sérologique vis-à-vis du CMV.

En revanche, les indications suivantes ne font pas l'objet d'un Accord professionnel :

- patients CMV négatifs en situation d'attente de greffe, afin de préserver leurs chances de rester CMV négatifs ;

- receveurs CMV négatifs de greffe autre que celles de cellules souches hématopoïétiques ou de poumon, lorsque le donneur est CMV positif ;

- receveurs de greffe CMV positifs.

\section{Transfusion de globules rouges en cas d'urgence hémorragique, d'anesthésie ou de réanimation}

\subsection{Anémie aiguë : généralités}

Elle recouvre essentiellement les anémies par hémorragies.

La nécessité de transfuser des GR repose sur le besoin d'augmenter le transport artériel de l' $\mathrm{O}_{2}$ aux tissus.

$$
\begin{aligned}
& \text { Rappel physiologique } \\
& \dot{\mathrm{T}}_{\mathrm{aO}}=\dot{\mathrm{Q}} \times \mathrm{CaO}_{2} \approx \dot{\mathrm{Q}} \times \mathrm{SaO}_{2} \times[\mathrm{Hb}] \times 1,39
\end{aligned}
$$

Le seuil critique $\left(\dot{\mathrm{TaO}}_{2}\right.$ crit) chez l'homme anesthésié est de l'ordre de $5 \mathrm{~mL} \mathrm{O} \cdot \mathrm{kg}^{-1} \cdot \mathrm{min}^{-1}$ (grade $B$ ). Pour conserver une

\begin{tabular}{|c|c|}
\hline Transformation & Indications \\
\hline Déleucocytation & Tous les PSL depuis le $1^{\mathrm{er}}$ avril 1998 \\
\hline \multirow{2}{*}{ Déplasmatisation } & Intolérance aux protéines plasmatiques \\
\hline & Antécédents de purpura post-transfusionnel \\
\hline \multirow[t]{3}{*}{ Cryoconservation } & $\begin{array}{l}\text { Mêmes indications que pour la déplasmatisation } \\
\text { plus }\end{array}$ \\
\hline & Phénotype érythrocytaire rare \\
\hline & Patient poly-immunisé \\
\hline \multirow[t]{7}{*}{ Irradiation } & Déficit immunitaire congénital cellulaire \\
\hline & Avant et pendant un prélèvement de cellules souches hématopoïétiques autologues \\
\hline & $\begin{array}{l}\text { Patients traités par greffe de cellules souches hématopoïétiques autologues et allogéniques, pendant au moins } 1 \text { an } \\
\text { après autogreffe et à vie après allogreffe }\end{array}$ \\
\hline & Certaines polychimiothérapies anticancéreuses intensives \\
\hline & Transfusion intra-utérine \\
\hline & Exsanguino-transfusion et transfusion massive chez le prématuré \\
\hline & Dons dirigés intrafamiliaux (encadrés réglementairement) \\
\hline Qualification & Indications \\
\hline \multirow[t]{4}{*}{ Phénotypé } & Patients avec un ou plusieurs allo-anticorps érythrocytaires (réglementaire) \\
\hline & Patientes en âge de procréer (réglementaire) \\
\hline & Patients transfusés de façon itérative \\
\hline & Souhaitable pour tout patient ayant une espérance de vie raisonnable \\
\hline Compatibilisé & $\begin{array}{l}\text { Patients présentant ou ayant présenté ou suspecté de présenter un ou plusieurs allo-anticorps érythrocytaires } \\
\text { (réglementaire) }\end{array}$ \\
\hline CMV négatif & Allogreffes de cellules souches hématopoïétiques en situation où donneur et receveur sont CMV négatifs \\
\hline
\end{tabular}

Tableau III. Principales indications des produits transformés et qualifiés 
marge de sécurité suffisante, le seuil de sécurité chez l'adulte est situé à $10 \mathrm{~mL} \mathrm{O} 2 \cdot \mathrm{kg}^{-1} \cdot \mathrm{min}^{-1}$ (Accord professionnel).

Il découle de ces données physiologiques que la tolérance à l'anémie aiguë est fonction des possibilités d'augmentation du débit cardiaque, ce qui explique d'une part que la correction de l'hypovolémie est la première urgence, d'autre part que le seuil transfusionnel soit plus élevé chez les personnes atteintes d'insuffisance cardiaque. L'augmentation de la $\dot{\mathrm{VO}}_{2}$ (fièvre, agitation...) réduit la tolérance à l'anémie.

\section{Seuil transfusionnel}

La notion de seuil transfusionnel est critiquée, car la transfusion est une décision complexe, dans laquelle intervient notamment, outre la concentration en hémoglobine ([Hb]), la réserve cardiaque, l'estimation de la vitesse du saignement, la tolérance clinique. Toutefois, la notion selon laquelle il n'existe pratiquement pas de situation nécessitant une concentration supérieure à $10 \mathrm{~g} . \mathrm{dL}^{-1}$ n'est pas remise en cause. Un seuil de l'ordre de 7 g.dL ${ }^{-1}$ est généralement admis au cours de l'anesthésie des sujets sans antécédents cardiovasculaires.

Pour les patients ayant de tels antécédents, il n'existe pas de bénéfice démontré pour des concentrations supérieures à 8 g.dL ${ }^{-1}$. Il apparaît raisonnable de conserver le seuil de 10 g.dL $\mathrm{dL}^{-1}$ dans les cas de pathologie cardiaque avérée et menaçante.

En résumé, avec toutes les réserves que la notion de seuil impose, les seuils suivants sont retenus (Accord professionnel) :

- 7 g.dL $\mathrm{dL}^{-1}$ chez les personnes sans antécédents particuliers ;

- 8-9 g.dL $\mathrm{d}^{-1}$ chez les personnes ayant des antécédents cardiovasculaires ;

- $\quad 10$ g.dL $\mathrm{dL}^{-1}$ chez les personnes ne tolérant pas cliniquement les concentrations inférieures ou atteintes d'insuffisance coronaire aiguë ou d'insuffisance cardiaque avérée.

Ces valeurs ne sont pas applicables avant l'âge de 2 ans en raison de l'augmentation de la $\mathrm{TaO}_{2}$ : modification de l'affinité d'Hb pour l' $\mathrm{O}_{2}$, augmentation de la consommation de l'organisme en $\mathrm{O}_{2}$, diminution des capacités d'adaptation par réduc- tion des réserves inotropes du cœur. De plus, l'existence d'une détresse respiratoire peut être responsable d'une hypoxémie.

Indications en cas d'anémie aiguë (Accord professionnel)

- Etat de choc malgré la correction de l'hypovolémie.

- Persistance, malgré la correction de l'hypovolémie, des symptômes de mauvaise tolérance associés à une chute d'[Hb].

Les signes de gravité d'une anémie aiguë les plus fréquents sont : syncope, dyspnée, tachycardie, angor, hypotension orthostatique, accident ischémique transitoire.

La recherche des arguments cliniques doit intégrer les capacités d'adaptation du sujet à la baisse du transport en $\mathrm{O}_{2}$, importantes chez un sujet jeune en bonne santé, mais limitées chez le sujet âgé, insuffisant cardiaque ou respiratoire. Une coronaropathie connue ou asymptomatique constitue le principal facteur limitant l'augmentation du débit cardiaque et majore la morbidité et la mortalité.

Chez un sujet jeune en bonne santé, une polypnée excessive, une tachycardie supérieure à $130 \mathrm{~min}^{-1}$ ou une hypotension persistante doivent faire envisager la transfusion de CGR.

Chez un sujet âgé ou coronarien ou porteur d'un rétrécissement aortique, l'apparition ou l'aggravation d'un angor, des modifications (y compris asymptomatiques) de l'ECG en faveur d'une ischémie myocardique constituent une indication, de même que l'apparition d'un déficit neurologique (y compris transitoire) chez le sujet âgé ou vasculaire.

Chez un sujet insuffisant cardiaque ou respiratoire, une altération de la vigilance, une lipothymie d'effort ou une hypotension persistante incitent à la transfusion, de même qu'une baisse significative de la $\mathrm{PaO}_{2}$.

Les $\beta$-bloquants et les inhibiteurs calciques bradycardisants, qui limitent l'efficacité des mécanismes compensateurs, doivent être intégrés à la décision transfusionnelle.

Que le patient soit transfusé ou non, les symptômes de mauvaise tolérance, persistants ou témoins d'une récidive du saignement, doivent être recherchés régulièrement.

Tableau IV. Symptomatologie en fonction de l'importance des pertes sanguines chez l'adulte sans comorbidité

\begin{tabular}{|c|c|c|c|c|}
\hline & \multicolumn{4}{|l|}{ Perte sanguine (mL) } \\
\hline & 750 & $800-1500$ & $1500-2000$ & $>2000$ \\
\hline PA systolique & Inchangée & Normale & Diminuée & Très basse \\
\hline PA diastolique & Inchangée & Augmentée & Diminuée & Très basse ou imprenable \\
\hline Pouls $\left(\min ^{-1}\right)$ & Tachycardie modérée & $100-120$ & > 120 (faible) & > 120 (très faible) \\
\hline Recoloration capillaire & Normale & Lente (> $2 \mathrm{~s})$ & Lente (> $2 \mathrm{~s})$ & Indétectable \\
\hline Fréquence respiratoire & Normale & Normale & Tachypnée (> $\left.20 \mathrm{~min}^{-1}\right)$ & Tachypnée (> $\left.20 \min ^{-1}\right)$ \\
\hline Débit urinaire $\left(\mathrm{mL} \cdot \mathrm{h}^{-1}\right)$ & $>30$ & $20-30$ & $10-20$ & $0-10$ \\
\hline Extrémités & Normales & Pâles & Pâles & Pâles et froides \\
\hline Coloration & Normale & Pâle & Pâle & Grise \\
\hline Conscience & Normale & Anxiété ou agressivité & Anxiété ou agressivité ou altérée & Altérée ou coma \\
\hline
\end{tabular}

PA = pression artérielle. 
Tableau V. Classification des symptômes en fonction de l'importance des pertes sanguines chez l'enfant

Perte sanguine inférieure à $15 \%$ de la masse sanguine totale (Classe 1)

Pression artérielle normale

Fréquence cardiaque augmentée de 10 à $20 \%$

Perte sanguine comprise entre $\mathbf{2 0}$ et $\mathbf{2 5} \%$ de la masse sanguine totale (Classe 2)

Tachycardie $>150 \mathrm{~min}^{-1}$

Tachypnée > 35-40 $\mathrm{min}^{-1}$

Allongement du temps de recoloration cutanée

Diminution de la pression artérielle

Perte sanguine comprise entre 30 et $35 \%$ de la masse sanguine totale (Classe 3 )

Tous les signes précédents sont présents

Débit urinaire $<1 \mathrm{~mL} \cdot \mathrm{kg}^{-1} \cdot \mathrm{h}^{-1}$

Perte sanguine supérieure à $50 \%$ de la masse sanguine totale (Classe 4)

Pouls non palpables

Toute décision transfusionnelle et donnée de surveillance doivent être notifiées et étayées dans le dossier du patient.

\section{Volume à transfuser}

La transfusion est réalisée de préférence sur une veine périphérique.

Le nombre de CGR à transfuser $\left(\mathrm{N}_{\mathrm{CGR}}\right)$ dépend :

- de la quantité d'Hb présente dans chaque CGR $\left(\mathrm{QHb}_{\mathrm{CG}}\right)$;

- du volume sanguin total du patient (VST en mL) ;

- de la concentration initiale d' $\mathrm{Hb}\left(\mathrm{Hb}_{\mathrm{i}}\right.$ en $\left.\mathrm{g} . \mathrm{dL}^{-1}\right)$;

- de la concentration finale d'Hb désirée $\left(\mathrm{Hb}_{\mathrm{d}}\right.$ en $\left.\mathrm{g} \cdot \mathrm{dL}^{-1}\right)$.

$\mathrm{N}_{\mathrm{CGR}}=(\mathrm{VST} / 100)\left(\mathrm{Hb}_{\mathrm{d}}-\mathrm{Hb}_{\mathrm{i}}\right) / \mathrm{QHb} \mathrm{b}_{\mathrm{CG}}$

Le VST peut être calculé soit à l'aide d'abaques prenant en compte le poids et la taille, soit par la règle des 5 de Gilcher (tableau VI).

Chez l'enfant, le volume est calculé sachant que 3-4 $\mathrm{mL} . \mathrm{kg}^{-1} \mathrm{de}$ CGR élèvent [Hb] de $1 \mathrm{~g} . \mathrm{dL}^{-1}$.

Chez la femme enceinte ou chez un patient âgé de plus de 70 ans, aucune formule ne permet d'évaluer la quantité de CGR à transfuser, qui est la quantité minimale permettant la disparition des signes de mauvaise tolérance et/ou l'obtention d'une concentration acceptable d'Hb.

Chez l'adulte, la transfusion est effectuée sur la base d'un CGR pouvant être suffisant à faire disparaître les symptômes de mauvaise tolérance. L'attitude selon laquelle il ne convient pas
Pas de modification du temps de recoloration cutanée

Diminution de l'amplitude du pouls

Hypotension orthostatique $>10-15 \mathrm{mmHg}$

Débit urinaire $>1 \mathrm{~mL} \cdot \mathrm{kg}^{-1} \cdot \mathrm{h}^{-1}$

Somnolence, vomissements, sueurs, agitation

Obnubilation

de transfuser un seul CGR est obsolète. Selon la formule indiquée, il est attendu qu'un CGR augmente [Hb] de 1,4 g.dL ${ }^{-1}$ chez une femme de $50 \mathrm{~kg}$ et de $0,7 \mathrm{~g} . \mathrm{dL}^{-1}$ chez un homme de $90 \mathrm{~kg}$.

Vitesse de correction

Aucune étude ne permet de déterminer la vitesse optimale de correction, en particulier chez le coronarien.

Vitesse de perfusion habituellement utilisée :

- chez l'adulte : 10 à 15 mL.min ${ }^{-1}$, soit un CGR en 20 minutes ;

- chez le nouveau-né : 3 à $15 \mathrm{~mL} \cdot \mathrm{kg}^{-1} \cdot \mathrm{h}^{-1}$.

La vitesse maximale est limitée par :

- les effets délétères potentiels d'un apport massif de GR ;

- complications hémodynamiques, dominées par l'OAP ;

- hyperkaliémie secondaire à l'apport rapide de potassium

(n'a en pratique de conséquences qu'en pédiatrie ou chez l'insuffisant rénal, l'effet étant généralement minime chez l'adulte normal) ;

- hypothermie (le réchauffement des CGR par un matériel thermostaté est nécessaire) ;

- les moyens de perfusion utilisés. L'accélération de la transfusion peut être obtenue, dans l'ordre croissant d'efficacité : par gravité (élévation du flacon), par utilisation d'une tubulure à bulbe (blood pump), d'un manchon sous pression à gonflage manuel, d'une pompe à galets ayant un système de détection des bulles d'air avec alarme non neutralisable.

Tableau VI. Règle des 5 de Gilcher permettant de calculer le volume sanguin total (VST)

\begin{tabular}{lll}
\hline Femme & Obèse : $60 \mathrm{~mL} \cdot \mathrm{kg}^{-1}$ & Normale : $70 \mathrm{~mL} . \mathrm{kg}^{-1}$ \\
& Maigre $: 65 \mathrm{~mL} \cdot \mathrm{kg}^{-1}$ & Athlétique $: 75 \mathrm{~mL} . \mathrm{kg}^{-1}$ \\
Homme & VST de la femme $+5 \mathrm{~mL} . \mathrm{kg}^{-1}$ & \\
Enfant de plus de 1 an & Identique à la femme adulte normale $\left(70 \mathrm{~mL} . \mathrm{kg}^{-1}\right)$ & \\
Nourrisson de moins de 1 an & $70-75 \mathrm{~mL} . \mathrm{kg}^{-1}$ & \\
Nouveau-né & $75-85 \mathrm{~mL} . \mathrm{kg}^{-1}$ & \\
\hline
\end{tabular}




\subsection{Anémie aiguë au cours d'un acte chirurgical}

Il n'existe pas de consensus sur le niveau acceptable d'hémodilution. Des concentrations relativement basses en $\mathrm{Hb}$ sont souvent bien tolérées sous anesthésie. L'importance constatée du saignement peut justifier la transfusion d'emblée.

\subsection{En pré-opératoire}

En présence de signes pré-opératoires de mauvaise tolérance, que l'anémie soit aiguë ou chronique, la situation ne se distingue pas de ce qui est indiqué par ailleurs. Il n'existe pas de données permettant de privilégier de principe une transfusion pré-opératoire. L'état supposé des coronaires et de la fonction ventriculaire gauche sont les principaux facteurs de décision.

En l'absence de signes pré-opératoires de mauvaise tolérance, il n'existe pas de donnée justifiant une modification du seuil transfusionnel en cas d'intervention mineure non hémorragique, du seul fait de l'intervention ou de l'anesthésie.

\subsubsection{En per-opératoire}

La transfusion est fréquemment nécessaire quand [Hb] préopératoire $<8 \mathrm{~g}$. $\mathrm{dL}^{-1}$ et le saignement per-opératoire est supérieur à $500 \mathrm{~mL}$.

Une $[\mathrm{Hb}]$ de l'ordre de 7 g.dL ${ }^{-1}$ est généralement bien tolérée chez le sujet jeune sans pathologie médicale ou anémie chronique bien compensée. Il est recommandé de surveiller régulièrement l'hématocrite ou, mieux, l'hémoglobine au cours d'une chirurgie hémorragique. Chez le sujet atteint de pathologie cardiaque ou si $[\mathrm{Hb}]<7$ g.dL ${ }^{-1}$, le monitorage de la $\mathrm{SvO}_{2}$ peut être recommandé. Chez le coronarien, une détection de l'ischémie myocardique par surveillance du segment ST est recommandée.

\subsubsection{En post-opératoire}

D'une part la contrainte métabolique est plus élevée, d'autre part la surveillance est généralement moins rapprochée qu'au bloc opératoire, au fur et à mesure que le temps passe.

Seuil transfusionnel recommandé : $[\mathrm{Hb}]=8 \mathrm{~g} \cdot \mathrm{dL}^{-1}$. Il n'y a pas d'étude montrant une diminution de la morbidité ou de la mortalité pour des $[\mathrm{Hb}]>8 \mathrm{~g} \cdot \mathrm{dL}^{-1}$, y compris chez les patients de réanimation (grade $\mathrm{B}$ ).

En fait, la décision finale est clinique. Schématiquement, deux attitudes sont possibles :

- soit adapter les traitements et la surveillance au niveau d'hémodilution ;

- $\quad$ soit adapter le niveau d'hémodilution aux possibilités de la thérapeutique et de la surveillance.

L'absence de frisson obtenu par le maintien de la normothermie ou la poursuite de l'anesthésie, et la surveillance en soins intensifs peuvent autoriser des taux d'hémodilution comparables à ceux du peropératoire, lorsque l'évolution reste non compli- quée. A l'opposé, la prévision d'un frisson ou d'une fièvre, d'une agitation, une surveillance ordinaire en salle peuvent faire préférer une concentration plus élevée d'Hb (Accord professionnel).

Dans le cas de l'infarctus du myocarde à la phase aiguë, de l'angor instable et de l'insuffisance ventriculaire gauche, il apparaît raisonnable de rester au-dessus du seuil de 10 g.dL ${ }^{-1}$. Chez le coronarien en dehors de toute pathologie aiguë, il n'y a pas d'argument pour recommander un seuil d' $[\mathrm{Hb}]>8$ g.dL ${ }^{-1}$. La transfusion est adaptée au débit du saignement observé, de façon à maintenir $[\mathrm{Hb}]>$ seuil (Accord professionnel). Une détection de l'ischémie myocardique par surveillance du segment ST est recommandée.

Ces recommandations sont également valables pour la femme enceinte et les enfants âgés de plus de 2 ans.

Les méthodes d'épargne de sang homologue sont traitées dans le chapitre 6.

\subsection{Produits transfusés en cas d'urgence}

Le conflit entre l'intérêt de transfuser sans délai préjudiciable et celui de disposer des produits correspondant aux qualifications jugées utiles, oblige à préciser le degré d'urgence, à organiser la distribution afin de faciliter l'accès aux produits les plus adaptés et à formaliser un algorithme décisionnel (Annexe III).

La distribution de CGR sera réalisée, avec des produits dont la qualification est la plus proche possible du phénotype érythrocytaire du patient. Elle tiendra compte :

- des femmes en âge de procréer ;

- des patients ayant des allo-anticorps anti-érythrocytaires ;

- des sujets susceptibles d'être retransfusés, ayant une espérance de vie raisonnable (cf. chapitre 1.2).

Les qualifications autres qu'immuno-hématologiques et les transformations ne seront prises en compte que s'il existe des produits disponibles correspondant exactement aux besoins des patients.

\subsubsection{Urgence vitale immédiate}

L'obtention des PSL doit être la plus rapide possible et la distribution sans délai. Les CGR seront éventuellement distribués (même si des résultats valides ne sont pas disponibles) sans groupe sanguin et sans recherche d'agglutinines irrégulières (RAI) : O RH : -1 , KEL : -1 , voire O RH : 1 (si possible RH : $-3,-4)$ KEL : -1 (anciennement $\mathrm{O}$ Rh D nég, KELL nég voire O Rh D pos [si possible RH E-, c-] KELL nég) et dépourvus d'hémolysine. En présence de données valides d'immunohématologie et en contexte d'urgence, il est recommandé de distribuer des CGR de groupe KEL : -1 (KELL nég) dans tous les cas, RH : -1 (Rh D nég) si le phénotype du patient est 
R : -1 (Rh D nég), RH : 1 (Rh D pos) (RH : $-3,-4$ [anciennement E-,c-]) si le phénotype du patient est $\mathrm{RH}: 1$ (Rh D pos). La prescription des PSL mentionnera l'urgence vitale immédiate et sera accompagnée des échantillons pour les analyses immuno-hématologiques dès que possible.

\subsubsection{L'urgence vitale}

L'obtention des PSL doit être inférieure à 30 minutes. Les CGR doivent être distribués avec un groupe conforme, éventuellement sans RAI si l'examen n'est pas disponible. La prescription des PSL mentionnera l'urgence vitale et sera accompagnée des échantillons pour les analyses immuno-hématologiques. La RAI sera réalisée dès que possible.

\subsubsection{L'urgence " relative "}

Le temps disponible est le plus souvent de 2 à 3 heures, ce qui permet la réalisation de l'ensemble des examens immunohématologiques (dont la RAI si elle date de plus de 3 jours); les PSL distribués seront isogroupes, au besoin compatibilisés. La situation hémorragique pouvant se modifier à tout moment, il sera possible de re-qualifier le degré d'urgence.

Dans le cadre de l'urgence, il n'existe pas aujourd'hui d'alternative à la transfusion de GR.

\section{Transfusion de globules rouges en cas d'anémie chronique}

\subsection{Généralités}

La durée d'évolution de l'anémie, permettant de la définir comme « chronique », n'est pas établie.

Indications en cas d'anémie chronique

- Correction des symptômes associés à une diminution $\mathrm{d}^{\prime}[\mathrm{Hb}]$.

Les symptômes d'anémie chronique sont : asthénie, irritabilité, palpitations, dyspnée d'effort, céphalées et vertiges.

Le caractère subjectif et non spécifique explique probablement l'absence de corrélation avec $[\mathrm{Hb}]$. La tolérance clinique est très variable d'un individu à l'autre et varie selon son activité physique.

Une transfusion n'est indiquée qu'en l'absence de traitement étiologique disponible (traitement d'une carence en fer, folates, vitamine $\mathrm{B}_{12}$, arrêt d'un médicament hématotoxique lorsque c'est possible, traitement d'une maladie inflammatoire) ou lorsque la sévérité de l'anémie ne permet pas d'attendre la réponse à ce traitement étiologique.

Au cours d'une anémie chronique, l'indication d'une transfusion de CGR doit être discutée en fonction :
- de sa sévérité, jugée sur l'hémoglobinémie et la tolérance clinique, en tenant compte des mécanismes compensateurs du patient (adaptabilité du débit cardiaque en particulier) ;

- de sa cause et de son mode d'installation ;

- du rapport bénéfice/risques de la transfusion pour le patient. Les méthodes d'épargne de sang homologue sont traitées dans le chapitre 6.

Seuil transfusionnel

$-[\mathrm{Hb}]=10 \mathrm{~g} \cdot \mathrm{dL}^{-1}:$ les indications sont rares et restreintes aux patients atteints de pathologie cardiopulmonaire manifestant des signes d'intolérance.

$-[\mathrm{Hb}]=8 \mathrm{~g} \cdot \mathrm{dL}^{-1}:$ les indications sont restreintes aux patients devant être actifs et limités dans leur activité, ainsi qu'aux personnes ayant des antécédents cardiovasculaires.

$-[\mathrm{Hb}]=6 \mathrm{~g} \cdot \mathrm{dL}^{-1}:$ la transfusion est généralement indiquée, sauf en cas de bonne tolérance (anémie de Biermer, anémies ferriprives, certaines anémies hémolytiques chroniques, anémie de l'insuffisance rénale chronique).

Les indications des transfusions en fonction de l'âge et du terrain sont mal définies. Chez le sujet âgé, $[\mathrm{Hb}]$ doit être interprétée en fonction de l'état d'hydratation et d'une éventuelle insuffisance rénale fonctionnelle. En cas de trouble de l'hydratation, celui-ci mérite d'être corrigé au préalable, ce qui permet de mieux apprécier $[\mathrm{Hb}]$.

Volume à transfuser

Il n'existe pas d'autre mode de calcul que celui défini pour l'anémie aiguë. Chez les personnes âgées ou insuffisantes cardiaques, la transfusion se fait sur la base d'un seul CGR à la fois (Accord professionnel).

Vitesse de correction

Elle doit être lente pendant les 15 premières minutes $(\leq 5$ $\mathrm{mL} \cdot \mathrm{min}^{-1}$ ), puis peut être augmentée (jusqu'à $10 \mathrm{~mL} \cdot \mathrm{min}^{-1}$ ) en l'absence de signes cliniques d'intolérance. En cas de surcharge volémique, notamment chez l'insuffisant cardiaque, elle reste lente durant toute la transfusion $\left(\leq 5 \mathrm{~mL} \cdot \mathrm{min}^{-1}\right)$; la position demiassise et l'emploi de diurétiques peuvent alors être indiqués.

\subsection{Cas particuliers}

\subsubsection{Personnes âgées}

Les signes cliniques peuvent être atypiques : malaise, chute, confusion, etc. Chez les personnes âgées, les mécanismes compensateurs, cardiaque et vasomoteur périphérique, sont fréquemment altérés.

Si l'espérance de vie est courte, les risques à long terme de la transfusion sont à mettre en balance avec le bénéfice à court terme. 
Prenant en compte le risque cardiovasculaire, il est habituel de ne pas descendre le seuil transfusionnel au-dessous de 8 g.dL ${ }^{-1}$.

La quantité de CGR à transfuser et la vitesse de transfusion doivent tenir compte du risque de surcharge volémique et de ses conséquences (OAP), notamment en cas d'insuffisance cardiaque préexistante.

\section{2 .2 Grossesse}

En raison de l'augmentation du volume plasmatique, [Hb] diminue en fin de grossesse de 5 à $10 \%$.

Le risque de retentissement fœtal est à prendre en compte à partir d'une $\mathrm{Hb}<9$ g.dL ${ }^{-1}$. Toute anémie doit être explorée. Le plus souvent, il s'agit d'une carence en fer ou en folates, facilement corrigeable et que l'on peut prévenir durant le dernier trimestre de la grossesse.

\subsubsection{Pathologies cardiaques}

Toute baisse d'[Hb] peut engendrer ou aggraver une ischémie myocardique. La majorité des manifestations coronariennes d'origine anémique paraît se manifester chez des sujets atteints d'une pathologie coronarienne sténosante. L'anémie chronique est généralement bien tolérée chez l'insuffisant cardiaque.

\subsubsection{Autres pathologies}

Aucun travail rigoureux n'a été mené chez les patients anémiques, en particulier âgés, ayant une insuffisance respiratoire chronique, une pathologie artérielle, en particulier périphérique, ou une affection cérébrale vasculaire ou dégénérative, permettant de définir les indications optimales éventuelles des transfusions de CGR.

\section{Transfusion de globules rouges en hématologie et en oncologie}

\subsection{Transfusion de globules rouges au cours des hémopathies malignes et en oncologie}

\subsubsection{Au cours des hémopathies malignes aiguës de l'adulte} et des greffes de cellules souches hématopoḯtiques Indication au cours d'hémopathies malignes aiguës

Elle doit tenir compte de la cinétique de l'aplasie induite par la chimiothérapie et de la date attendue de la sortie d'aplasie. Le maintien d'[Hb] supérieure au seuil fixé est d'autant plus à respecter que la date prévisible de la sortie d'aplasie est plus éloignée.

Chez les patients en fin de vie, aucune étude n'est disponible concernant les indications des CGR. Il est important d'étudier au cas par cas le retentissement objectif, mais aussi subjectif de l'anémie et le mieux-être réellement apporté par les transfusions, afin d'éviter une prescription systématique fondée sur la seule $[\mathrm{Hb}]$. Ce bénéfice doit être réévalué après chaque transfusion et comparé aux éventuels effets indésirables et aux simples contraintes (importance des réactions transfusionnelles chez les malades poly-immunisés, déplacements imposés pour la réaliser, etc.).

Seuil transfusionnel

Seuil recommandé : $[\mathrm{Hb}]=8 \mathrm{~g} \cdot \mathrm{dL}^{-1}$, lorsque la correction spontanée de l'anémie n'est pas prévisible à court terme (Accord professionnel).

Il peut toutefois être plus élevé, de l'ordre de 9-10 g.dL ${ }^{-1}$, dans les circonstances qui augmentent la consommation d' $\mathrm{O}_{2}$ : infections sévères, bronchospasmes, complications pulmonaires, cardiaques réduisant la réserve fonctionnelle cardiaque (ischémie myocardique, fibrillation auriculaire).

Produits transfusés

- CGR déleucocytés, phénotypés au moins dans le phénotype RH et KELL, et irradiés (dans les indications figurant dans le tableau III).

Dans le cas particulier des allogreffes, les règles transfusionnelles tiennent compte des groupes érythrocytaires du donneur et du receveur et du délai d'administration des concentrés par rapport à la date de greffe. Pour l'allogreffe de cellules souches hématopoḯtiques en situation non apparentée les produits CMV négatifs restent recommandés.

\subsubsection{Au cours des hémopathies malignes chroniques et en} oncologie chez l'adulte

Indications au cours d'hémopathies malignes chroniques et en oncologie

- Indications et modalités de transfusion identiques à celles des hémopathies malignes aiguës chez les patients qui reçoivent une chimiothérapie intensive avec greffe de cellules souches hématopoiétiques.

- Dans toutes les autres situations, l'anémie chronique est une complication habituelle et multifactorielle, surtout en phase avancée ou après des chimiothérapies intensives. Elle peut être temporaire et ne pas nécessiter de transfusion systématique, notamment chez les patients jeunes. Elle peut persister et s'aggraver du fait de l'évolution de la maladie et de la toxicité des traitements employés. Il peut alors être nécessaire de la corriger de façon prolongée, ce d'autant qu'un impact négatif de l'anémie sur l'efficacité de la radiothérapie a été suggéré, notamment dans les cancers ORL et utérins.

Seuil transfusionnel

Seuil recommandé : celui de la disparition des symptômes liés à l'anémie, sans généralement descendre sous le seuil de 8 g. $\mathrm{dL}^{-1}$ (Accord professionnel). 
Transfuser au-delà de ce seuil expose le patient aux complications de l'hypertransfusion lorsque son pronostic laisse espérer une survie de plusieurs années.

Ce seuil peut être plus élevé ( 10 g.dL $\left.\mathrm{dL}^{-1}\right)$ en cas d'anémie mal tolérée, en particulier en cas de dyspnée au cours de cancer bronchique ou de métastases pulmonaires symptomatiques, notamment chez les patients âgés (Accord professionnel).

Les méthodes d'épargne de sang homologue sont traitées dans le chapitre 6 .

\subsubsection{En oncologie pédiatrique}

Indication en oncologie pédiatrique

- Anémie symptomatique (Accord professionnel). La tolérance de l'anémie est bonne chez l'enfant et sa correction est le plus souvent non urgente.

\section{Seuil transfusionnel}

Seuil recommandé : $[\mathrm{Hb}]=8 \mathrm{~g} \cdot \mathrm{dL}^{-1}$ (seuil pour lequel il existe des arguments physiopathologiques chez l'adulte) chez un enfant recevant une chimiothérapie continue ne laissant pas espérer une correction spontanée à court ou moyen terme de son anémie.

Les méthodes d'épargne de sang homologue sont traitées dans le chapitre 6.

Produits transfusés

- CGR déleucocytés phénotypés. Le phénotypage est justifié chez les enfants de sexe féminin et par extension chez tous les enfants du fait de l'âge et du risque d'allo-immunisation pouvant avoir un impact à long terme (polytransfusions probables). Les indications de l'irradiation sont les mêmes que chez l'adulte, mais tendent à être élargies dans le but d'éviter au maximum la survenue d'une GVH post-transfusionnelle (Accord profession$n e l)$.

\section{Volume à transfuser}

Il doit être suffisant pour remonter [Hb] à un niveau susceptible de retarder une nouvelle transfusion, dans le souci de diminuer le nombre de donneurs. L'autre approche visant à diminuer le risque transfusionnel est l'utilisation, chez l'enfant de faible poids, de préparations pédiatriques.

La formule définie concernant l'anémie aiguë peut être utilisée, mais la formule suivante est couramment employée en onco-hématologie pédiatrique pour estimer le volume à transfuser :

Volume à transfuser $(\mathrm{en} \mathrm{mL}$ de $\mathrm{CGR})=\mathrm{P} \times \Delta \mathrm{Hb} \times(3$ à 4$)$

( $\mathrm{P}$ : poids en $\mathrm{kg} ; \Delta \mathrm{Hb}$ : élévation souhaitée d' $[\mathrm{Hb}]$ en $\left.\mathrm{g} \cdot \mathrm{dL}^{-1}\right)$.

\subsection{Transfusion de globules rouges au cours des hémoglobinopathies}

\subsection{1 $\beta$-Thalassémies homozygotes majeures (" anémie} de Cooley ")

Ces patients ayant une production spontanée d'Hb très faible, les apports transfusionnels conditionnent le pronostic vital.

Seuil transfusionnel

Seuil recommandé chez l'enfant et l'adolescent : $[\mathrm{Hb}]=10$ g.dL ${ }^{-1}$, seuil qui permet des activités scolaires, ludiques ou professionnelles normales et de réduire les troubles du développement et l'hyperplasie érythroïde responsable de déformations morphologiques. Les risques immunologiques, infectieux et de surcharge en fer liés à la transfusion ont conduit à ne pas choisir un seuil plus élevé. Les transfusions sont de $15 \mathrm{~mL} \cdot \mathrm{kg}^{-1}$ toutes les 3 semaines ou de $20 \mathrm{~mL} \cdot \mathrm{kg}^{-1}$ toutes les 4 semaines.

Le seuil transfusionnel peut être moins élevé chez l'adulte : 8 à 9 g.dL ${ }^{-1}$.

\section{Consommation annuelle}

Une consommation annuelle de l'ordre de 150-200 mL. $\mathrm{kg}^{-1}$ par an de CGR-SAGM maintient usuellement une [Hb] moyenne proche de $12 \mathrm{~g} \cdot \mathrm{dL}^{-1}$. Une consommation supérieure à $200 \mathrm{~mL} . \mathrm{kg}^{-1}$ par an doit faire rechercher la cause de l'inefficacité transfusionnelle, souvent due à un hypersplénisme, qui fait discuter la splénectomie.

Produits transfusés

- CGR déleucocytés phénotypés RH-KELL : avant la première transfusion, il est recommandé de pratiquer un phénotypage étendu (RH, KELL, Kidd, Duffy, MNS, etc.). L'utilisation de GR jeunes (néocytes) double le nombre de donneurs et n'est donc pas recommandée bien qu'elle permette d'allonger l'espace entre deux transfusions.

\subsubsection{Thalassémies homozygotes intermédiaires}

Ces patients ont spontanément une production d'Hb de 7 à 10 g.dL ${ }^{-1}$.

Indications au cours de thalassémie homozygote intermédiaire

- Aggravation de l'anémie chronique : elle peut être aiguë (infection, érythroblastopénie) et nécessite alors la transfusion. Elle peut être progressive et traduire un hypersplénisme ; la splénectomie réduit, voire supprime, les besoins transfusionnels.

- Signes d'intolérance de l'anémie chronique : fatigabilité, retentissement scolaire ou professionnel, retard pubertaire...

Des transfusions peuvent être nécessaires au cours de la grossesse.

\subsubsection{Drépanocytose homozygote}

On rapproche des drépanocytaires homozygotes les patients ayant des syndromes drépanocytaires de sévérité analogue (pa- 
tients hétérozygotes $\mathrm{S} \beta$-thalassémiques) ou de moindre sévérité (patients hétérozygotes $\mathrm{SC}$ ).

Chez tous ces patients une $[\mathrm{Hb}]$ de $8 \pm 1 \mathrm{~g} \cdot \mathrm{dL}^{-1}$ permet une activité et une croissance normales. Il n'y a pas nécessité de transfuser un patient drépanocytaire bien portant avec $[\mathrm{Hb}] \geq 6 \mathrm{~g} \cdot \mathrm{dL}^{-1}$.

Indications de la transfusion sanguine simple

La transfusion a pour but de ramener l'hémoglobinémie à sa valeur de base. Une diminution d'Hb par rapport à la concentration de base peut relever de plusieurs mécanismes :

- Hyperhémolyse provoquée par une infection quelconque ou contemporaine d'une crise vaso-occlusive : l'indication transfusionnelle se discute en fonction de la tolérance clinique et de l'amplitude de la réponse réticulocytaire.

- Infection à parvovirus B19 : la réticulocytopénie conduit au diagnostic. La transfusion est le plus souvent nécessaire.

- Une diminution transitoire de l'érythropoï̀se contemporaine d'un syndrome inflammatoire aigu : l'indication transfusionnelle se discute en fonction de la tolérance clinique et de la rapidité de la réascension de la concentration de réticulocytes.

- Séquestration splénique : elle peut être aiguë : le diagnostic repose sur une splénomégalie rapidement croissante alors qu'apparaît une déglobulisation brutale. C'est une urgence transfusionnelle. La séquestration peut devenir chronique, entraînant des besoins transfusionnels répétés; la splénectomie doit alors être discutée.

\section{Produits transfusés}

- CGR déleucocytés et phénotypés. Avant la première transfusion, il est recommandé de pratiquer un phénotypage étendu (RH, KELL, Kidd, Duffy, MNS, etc.). On peut le plus souvent se limiter aux systèmes RH et KELL, car le risque d'allo-immunisation dans les autres systèmes apparaît faible chez les drépanocytaires. L'apparition d'un allo-anticorps doit conduire à étendre la compatibilité phénotypique des CGR transfusés.

\section{Indications de l'échange transfusionnel ponctuel}

Il s'agit d'un acte transfusionnel associant une saignée et une transfusion, dont l'objectif est de diminuer la concentration en $\mathrm{HbS}$ tout en n'augmentant pas ou peu l'Ht. Il est parfois prescrit en urgence lors d'une complication vaso-occlusive grave ou de façon programmée pour préparer un patient à un acte chirurgical.

La survenue d'un syndrome thoracique aigu, d'un AVC, d'un priapisme, d'une séquestration hépatique sont une indication (Accord professionnel).

Cette indication peut être étendue aux chocs septiques et aux crises douloureuses résistantes aux antalgiques.

Le risque de complications post-opératoires, infectieuses ou vaso-occlusives, est élevé chez les patients drépanocytaires. La pratique habituelle est de procéder à un échange transfusionnel préopératoire abaissant $\mathrm{HbS}$ en dessous de 30-40\%, attitude qu'il est recommandé de moduler selon la durée de l'anesthésie et le type d'intervention.

Indications de l'échange transfusionnel au long cours

Des programmes d'échanges transfusionnels maintenant en permanence le taux d'HbS en dessous 30-40\% sont proposés chez certains patients drépanocytaires : après un AVC, lors d'une détérioration viscérale sévère (insuffisance respiratoire, rénale, cardiaque).

Chez la femme enceinte, une pratique fréquente est de transfuser entre le cinquième et le neuvième mois, afin de maintenir [Hb] entre 10 et 11 g.dL ${ }^{-1}$.

\subsubsection{Autres hémoglobinopathies}

Les autres hémoglobinopathies ne sont qu'exceptionnellement responsables d' anémie requérant des transfusions de CGR : citons l'homozygotie pour l'Hb C, E, certaines $\mathrm{Hb}$ instables.

4.2.5 Anomalies de la membrane du globule rouge et déficits en une enzyme érythrocytaire

La grande majorité des patients porteurs d'anomalies de la membrane érythrocytaire et de déficits enzymatiques érythrocytaires (en G6PD et en pyruvate kinase principalement) ne reçoit des CGR phénotypés RH-KELL et déleucocytés que lors des épisodes de déglobulisation aiguë. De rares patients (en particulier porteurs de déficit en pyruvate kinase) sont soumis à des programmes de transfusions itératives dont les modalités sont identiques à celles des patients thalassémiques.

4.3 Transfusion de globules rouges au cours des maladies constitutionnelles et de l'aplasie congénitale

\subsubsection{Au cours des maladies constitutionnelles et des aplasies} médullaires congénitales

Il s'agit d'anémies chroniques de l'enfant, le plus souvent bien tolérées.

Seuil transfusionnel

Seuil recommandé : $[\mathrm{Hb}]=7$ à $8 \mathrm{~g} \cdot \mathrm{dL}^{-1}$, en fonction de la tolérance clinique. Il n'existe aucune étude définissant un seuil optimal. Il n'y a pas d'indication à l'hypertransfusion.

Produits transfusés

- CGR phénotypés RH-KELL et déleucocytés.

Le phénotypage du patient doit être le plus étendu possible, réalisé avant la première transfusion et les donneurs strictement sélectionnés en fonction de ce phénotype.

Le statut CMV du receveur et du donneur n'est pas à prendre en compte chez ces enfants non immunodéprimés, par ailleurs protégés par la déleucocytation des CGR. L'irradiation n'est pas justifiée sur ce terrain non immunodéprimé. Les seules exceptions sont le don intrafamilial (obligation réglementaire) et la 
période se situant immédiatement avant la greffe de cellules souches hématopoïétiques.

\subsubsection{Au cours des aplasies médullaires acquises}

La transfusion de CGR obéit aux mêmes règles qu'en cas d'aplasie congénitale.

Des cas de GVH post-transfusionnelle ayant été rapportés, l'aplasie médullaire ne constitue une indication d'irradiation des CGR, ni chez l'adulte, ni chez l'enfant. Il existe néanmoins deux situations dans lesquelles l'irradiation est à considérer :

- en cas de traitement immunosuppresseur intense ;

- si une greffe de cellules souches hématopoïétiques est programmée rapidement.

\subsection{Transfusion de globules rouges au cours des} anémies hémolytiques acquises (AHA)

Indications au cours des AHA

- Formes aiguës graves d'AHA ou aggravation des formes chroniques.

- Formes chroniques sévères (plus rarement).

Au cours des AHA, comme dans toutes les anémies, la thérapeutique transfusionnelle doit être fondée sur de multiples éléments cliniques et biologiques : la concentration en $\mathrm{Hb}$, la rapidité d'installation de l'anémie, l'âge du patient, les pathologies associées, une éventuelle étiologie médicamenteuse, les maladies sous-jacentes éventuellement responsables de l'hémolyse et dont le traitement peut influencer cette dernière, la surveillance de l'évolution, l'appréciation des chances d'efficacité et des dangers de l'apport de GR. Il en est de même pour le choix de ses modalités (type de produit, quantité, température des produits à administrer).

Seuil transfusionnel

Ce qui prime, c'est la façon dont l'anémie est supportée.

Seuil recommandé : $[\mathrm{Hb}]=8 \mathrm{~g} . \mathrm{dL}^{-1}$, en cas d'anémie chronique mal tolérée, qui ne peut être corrigée autrement que par transfusion. On peut ne pas transfuser à des seuils inférieurs, notamment si on espère une amélioration à court terme, spontanée ou secondaire au traitement étiologique de l'AHA.

\section{Produits transfusés}

- CGR déleucocytés et phénotypés dans les systèmes RH et KELL.

Le clinicien doit connaître les difficultés pour réaliser les explorations sérologiques et mettre à disposition les produits sanguins adéquats, spécialement dans les AHA de mécanisme immunologique. Dans certains cas, il faut recourir à des laboratoires d'immuno-hématologie hautement spécialisés, et rechercher des produits érythrocytaires rares, parfois au plan national (Centre national de référence pour les groupes sanguins).
Il est le plus souvent impossible de transfuser des GR non porteurs d'antigènes reconnus par les auto-anticorps présents, car ceux-ci sont généralement dirigés contre des antigènes dits «publics », majoritairement présents dans la population. En cas d'auto-anticorps de spécificité restreinte, il est parfois possible de transfuser des GR dépourvus de l' antigène reconnu. Dans tous les autres cas, la survie des GR transfusés est abrégée, et la transfusion est d'effet médiocre, sans être vraiment dangereuse, malgré la démonstration in vitro de l'incompatibilité, liée à la présence des auto-anticorps. Les GR transfusés sont le plus souvent détruits par les auto-anticorps à la même vitesse (et même parfois plus rapidement) que ceux du patient.

Le risque majeur est la survenue d'accidents hémolytiques transfusionnels très graves du fait de l'absence de détection d'allo-anticorps acquis, masqués par les auto-anticorps. La recherche des allo-anticorps acquis nécessite des techniques sérologiques rigoureuses.

\subsubsection{Au cours des AHA auto-immunes}

\section{Indications au cours d'AHA auto-immunes}

La transfusion ne peut représenter au mieux qu'un apport substitutif temporaire, mais elle peut être le seul moyen de corriger un péril anoxique menaçant.

En dehors de sa faible efficacité et de la grande brièveté de ses effets, la transfusion peut être dangereuse, car responsable :

- d'accidents graves d'hémolyse immédiate due à la présence d'allo-anticorps immuns méconnus ou d'hémolyse retardée due à la réactivation de la synthèse d'allo-anticorps non détectables ;

- d'une allo-immunisation grevant l'avenir.

Elle peut également aggraver la situation : stimulation de la synthèse d'auto-anticorps, modifications du type des auto-anticorps, dont la spécificité pourrait devenir moins restreinte (mais l'inverse se produit aussi), passage d'un test de Coombs direct Ig à Ig + complément.

Le risque de la non-détection d'allo-anticorps acquis et la faible efficacité transfusionnelle ont conduit certains auteurs à prohiber toute transfusion de GR au cours des AHA auto-immunes, sauf menace vitale immédiate.

\section{Produits transfusés}

Le recours à des GR autologues congelés ne se fait qu'en cas d'AHA auto-immune caractérisée par des épisodes d'hémolyse à répétition, séparés par des périodes de disparition des auto-anticorps. Pendant ces périodes, les GR du malade pourraient être prélevés en vue de leur cryoconservation, à condition que :

- l'auto-anticorps soit strictement spécifique d'un antigène bien déterminé ; 
- ces GR (i) ne soient pas porteurs d'antigènes contre lesquels le malade possède déjà des allo-anticorps pouvant entraîner une hémolyse allo-immune ;

(ii) ne soient pas susceptibles de provoquer une alloimmunisation pouvant être ultérieurement dangereuse et compromettre l'avenir transfusionnel et obstétrical.

L'épreuve de compatibilité avec le sérum du receveur est presque toujours positive. Elle doit être faite à la température de fixation et d'activité des auto-anticorps détectés.

\section{Température des CGR transfusés}

En présence d'agglutinines froides ou d'anticorps biphasiques, il est recommandé de transfuser des GR portés à $37^{\circ} \mathrm{C}$, par un dispositif de réchauffement de la tubulure au lit du malade. La température de la pièce doit être éventuellement élevée audessus de celle à laquelle les auto-anticorps peuvent être nocifs.

\section{Surveillance de la transfusion}

Elle doit être extrêmement étroite. Il est prudent de surveiller particulièrement les dix premières minutes de la transfusion et, si l'état du patient le permet, de respecter à cette phase un débit de perfusion lent, de l'ordre de $2 \mathrm{~mL} \cdot \mathrm{min}^{-1}$. La surveillance biologique permet de s'assurer de l'efficacité de la transfusion par le dosage de l'Hb dans l'heure qui suit la fin de la transfusion. La surveillance biologique ultérieure comporte en particulier la recherche minutieuse et répétée de l'apparition d'allo-anticorps et de la non-modification de la spécificité des auto-anticorps.

Situations d'urgence vitale

Il faut transfuser les CGR nécessaires sans avoir la totalité des résultats des examens. Il est recommandé, et cela est pratiquement toujours possible, de réaliser avant la transfusion les prélèvements nécessaires à la détermination des groupes sanguins $\mathrm{ABO}$ et RH1 (Rh D) et à tous les examens immunohématologiques (en particulier pour l'analyse des auto-anticorps), et de réaliser les tests correspondants. L'urgence vitale autorise la transfusion de CGR avant les résultats de la recherche d'allo-anticorps, qui prend du temps en raison de la présence des auto-anticorps circulants.

4.4.2 Au cours des AHA immuno-allergiques

médicamenteuses, par agents chimiques,

agents physiques ou venins

En cas d'AHA immuno-allergique médicamenteuse, le médicament doit être arrêté (ce qui peut être difficile chez certains patients). Des agents divers peuvent provoquer des anémies aiguës parfois très sévères nécessitant des transfusions d'urgence, voire la réalisation d'exsanguino-transfusions.

Du point de vue transfusionnel, les mêmes problèmes se posent que dans les AHA auto-immunes : difficulté possible du groupage et de la recherche des allo-anticorps.

\subsubsection{Au cours de l'hémoglobinurie paroxystique nocturne}

Il est recommandé de recourir le moins possible à la transfusion de CGR. Ceux-ci sont déleucocytés et phénotypés ; ils ne sont plus déplasmatisés, sauf en cas d'inefficacité transfusionnelle faisant craindre la responsabilité de l'apport de complément par la transfusion.

\section{Transfusion de globules rouges en néonatalogie}

Les méthodes d'épargne de sang homologue sont traitées dans le chapitres 6 .

\subsection{Différents types de don de globules rouges}

\subsubsection{Donneur unique}

L'utilisation d'un donneur unique pour des transfusions multiples comporte deux avantages théoriques : immunologique et infectieux (réduction du nombre de donneurs).

Une poche de CGR est attribuée à chaque enfant pour une période de 42 jours. Lors de chaque transfusion, une poche de petit volume est préparée extemporanément à partir de ce CGR par l'ETS (transformation «préparation pédiatrique »).

\subsubsection{Don dirigé}

Le don dirigé n'est plus autorisé en France depuis le 4 janvier 1993, sauf nécessité thérapeutique (groupes sanguins exceptionnels, situations particulièrement complexes d'immunisation anti-érythrocytaire).

Lorsque le don parent-enfant est la seule possibilité, il faut vérifier la compatibilité entre le sérum du donneur et les GR de l'enfant. Le produit à transfuser doit être irradié et déplasmatisé en cas d'immunisation fœto-maternelle.

\subsubsection{Transfusion de CGR conservés moins de 7 jours}

Les CGR « frais » ont une durée de conservation inférieure à 7 jours. Ils contiennent des GR qui ont subi peu de lésions de stockage. La fraction de GR transfusés circulant 24 heures après transfusion est élevée (> $80 \%$ ) et décroît régulièrement jusqu'à une limite de 70-75\%, qui détermine le délai de péremption.

Indication à la transfusion de CGR conservés moins de 7 jours (Accord professionnel)

Chez le nouveau-né, les modifications métaboliques induites par la conservation au niveau des GR et du plasma étant redoutées, la transfusion de CGR conservés moins de 7 jours est recommandée en cas de :

- transfusion massive ( $\geq 1$ masse sanguine) en cas de perte volémique aiguë ;

- exsanguino-transfusion ;

- transfusions réalisées dans le cadre des techniques d'épuration extracorporelle du $\mathrm{CO}_{2}$. 
Dans toutes les autres indications, il est possible d'utiliser des CGR conservés plus de 7 jours sans effets néfastes chez le nouveau-né.

\subsection{Indications de la transfusion de globules rouges}

\subsubsection{Transfusion chez le fotus}

La transfusion intravasculaire nécessite un personnel expérimenté, mais apparaît plus performante que la transfusion intrapéritonéale, qui n'est réalisée que lorsque la transfusion intravasculaire est impossible, notamment à des termes très précoces.

\section{Indications chez le foetus}

- Anémies sévères liées aux allo-immunisations dans le système RH ou les autres systèmes de groupes sanguins.

- Anémie liée à une infection par le parvovirus B19 ou à une hémorragie foto-maternelle massive.

Elles sont déterminées en fonction de l' [Hb], de sa cinétique d'évolution (en cas de transfusions itératives), de la concentration d'anticorps présents dans le sang fotal, de l'importance de l'érythroblastose et de la réticulocytose fotales. Aucune étude ne valide un seuil à partir duquel les bénéfices de la transfusion apparaissent supérieurs à ses inconvénients.

Le but est de prolonger la durée de la grossesse en améliorant l'oxygénation tissulaire fœtale.

Les indications doivent tenir compte des risques inhérents à la transfusion fotale :

- risque interventionnel fotal, qui peut être évalué entre 1 et $3 \%$ en termes de létalité ;

- $\quad$ risque de réactivation de l'allo-imunisation foto-maternelle du fait de l'abord vasculaire fotal, notamment lorsqu'il est transplacentaire, car il peut mettre en communication les circulations fotale et maternelle ;

- risque infectieux post-transfusionnel pour la mère, qui peut être lié à l'injection de GR dans les lacs sanguins maternels, à une hémorragie fœto-maternelle ou à un saignement intraamniotique.

L'indication de prélèvement sanguin fœtal et la date de sa réalisation sont établies sur un faisceau d'arguments incluant :

- l'existence ou non d'œdèmes fœtaux, d'anasarque fœtoplacentaire ou de signes d'altération du bien-être fotal à l'échographie-Doppler ;

- le $\Delta$ de densité optique de la bilirubine à $450 \mathrm{~nm}$ ( $\Delta$ OD 450$)$ dans le liquide amniotique s'il est rapidement croissant (diagramme de Liley);

- une discordance entre la concentration sérique d'allo-anticorps maternels et les résultats du $\Delta$ OD 450 ;
- les antécédents obstétricaux et, en particulier, le terme auquel est survenue une éventuelle complication d'allo-immunisation lors d'une grossesse antérieure ;

- la nécessité d'obtenir le groupe sanguin fœtal (qui peut aussi être obtenu par PCR (réaction de polymérisation en chaîne) sur les cellules du liquide amniotique);

- l'existence d'anomalies du rythme cardiaque fotal évoquant une mauvaise tolérance de l'anémie du fotus.

Seuil transfusionnel

Il n'y a pas d'arguments pour privilégier l'un ou l'autre des seuils transfusionnels suivants :

- $\quad 10$ g.dL $\mathrm{dL}^{-1}$ (ou Ht fœtal < $30 \%$ ). Ce seuil est discutable car il ne tient pas compte de l'âge gestationnel ;

- 7 g.dL $\mathrm{dL}^{-1}$ au deuxième trimestre ou à 9 g.dL $\mathrm{dL}^{-1}$ au troisième trimestre ;

- -2 DS par rapport aux normes pour l'âge gestationnel.

Produits transfusés

Les CGR utilisés sont (Accord professionnel) :

- de moins de 5 jours, avec un contenu en $\mathrm{Hb}$ ou l'Ht (si possible autour de $80 \%$ ) connus ;

- de groupe $\mathrm{O}$, dépourvus d'hémolysine ;

- compatibilisés avec le sérum de la mère, en respectant impérativement l'antigénocompatibilité dans les systèmes RH et KELL, et éventuellement dans les autres systèmes reconnus immunogènes [Duffy, Kidd, MNS3 (S), MNS4 (s)] ;

- irradiés ;

- si possible CMV négatifs. Volume à transfuser

Pour une transfusion intravasculaire, le volume transfusionnel peut être déterminé sur le plan théorique par la formule :

Volume à transfuser $(\mathrm{en} \mathrm{mL})=$

$([\mathrm{Hb}]$ désirée $-[\mathrm{Hb}]$ fotale $) \times \mathrm{VSFP} /([\mathrm{Hb}]$ du produit $-[\mathrm{Hb}]$ désirée $)$

Volume sanguin fœto-placentaire (VSFP) $=90$ à 120 $\mathrm{mL} . \mathrm{kg}^{-1}$ de poids fœtal. On procède habituellement à une détermination pertransfusionnelle d'[Hb] du sang fotal lorsque les deux tiers du volume théorique ont été transfusés.

Objectif final : $[\mathrm{Hb}]=16$ à $17 \mathrm{~g} \cdot \mathrm{dL}^{-1} \mathrm{ou} \mathrm{Ht}$ fœtal $=45$ à $50 \%$. Des valeurs inférieures doivent être visées en cas d'anasarque fœto-placentaire. L'adéquation du volume transfusé est appréciée par les résultats des numérations sanguines fœtales réalisées en cours de transfusion.

Il n'existe pas d'argument objectif pour préconiser une exsanguino-transfusion in utero plutôt qu'une transfusion simple chez un fœtus anémique ne présentant pas de signe d'anasarque fœto-placentaire. A partir de la date de viabilité fœtale, une surveillance du bien-être fotal est nécessaire durant les heures qui suivent la transfusion in utero. 


\subsubsection{Transfusion chez le nouveau-né}

Il n'est pas recommandé de transfuser des CGR dans le seul but de maintenir une concentration en $\mathrm{Hb}$ (Accord professionnel).

L'indication de la transfusion de CGR dépend :

- de l'existence de signes cliniques d'intolérance de l'anémie ;

- du contexte pathologique et des facteurs de risque associés ;

- de l'importance de l'écart entre [Hb] et les valeurs de référence pour l'âge gestationnel et l'âge post-natal ;

- de la rapidité de survenue de l'anémie ;

- des possibilités de régénération médullaire.

Des études sont souhaitables pour valider des critères permettant d'apprécier la gravité de l'anémie chez les nouveau-nés et l'indication d'une transfusion de CGR.

En cas d'hémorragie aiguë, l'intensité et la durée de l'hémorragie, les possibilités d'en traiter la cause, la cinétique d'installation de l'anémie et son retentissement sur l'oxygénation tissulaire sont de meilleurs critères que la valeur isolée d'Ht ou d' [Hb].

Un collapsus dont l'origine n'est pas hémorragique n'est pas une indication reconnue de transfusion de CGR. Le maintien de la volémie doit être effectué par des solutions d'expansion volémique (grade A).

Chez le nouveau-né en détresse respiratoire sévère, en particulier s'il nécessite un support ventilatoire, le seuil de $[\mathrm{Hb}]=$ 12-13 g.dL ${ }^{-1}$ ou $\mathrm{Ht}=35-40 \%$ est habituellement recommandé, bien qu'il ait l'inconvénient de ne pas tenir compte de l'âge gestationnel, et qu'il ne soit pas validé par des études contrôlées (Accord professionnel).

La notion de seuil transfusionnel n'est pas définie chez le prématuré, passée la phase des complications vitales des premiers jours de vie, et chez le prématuré asymptomatique. Il est proposé de retenir à titre indicatif les seuils au-delà desquels une transfusion de GR n'est a priori pas indiquée :

- 12 g.dL $\mathrm{dL}^{-1}$ au cours de la période initiale des soins intensifs ;

- 10 g.dL $\mathrm{dL}^{-1}$ au cours de la période suivante des 2 premières semaines de vie ;

- 7 g.dL ${ }^{-1}$ et une concentration de réticulocytes de 100000 $\mathrm{mm}^{-3}$, ultérieurement.

\subsubsection{Exsanguino-transfusion chez le nouveau-né}

Indications de l'exsanguino-transfusion

- Maladie hémolytique, dans le but de soustraire des anticorps immuns dirigés contre les GR du nouveau-né, d'épurer la bilirubine libre et de corriger l'anémie. Son objectif est d'éviter l'ictère nucléaire et les autres formes de neurotoxicité de la bilirubine. L'exsanguino-transfusion améliore la survie des enfants présentant une allo-immunisation fœto-maternelle $\mathrm{RH}$ (grade B).

- D'autres indications ont été proposées : coagulations intravasculaires disséminées (CIVD), certaines erreurs innées du métabolisme de révélation néonatale. Le traitement optimal des causes de CIVD tend à en faire disparaître les indications. Le traitement des erreurs innées du métabolisme s'oriente vers des techniques d'hémodiafiltration.

Seuil d'exsanguino-transfusion

Le seuil d'indication est progressivement croissant avec l'âge post-natal jusqu' au troisième jour de vie. Il est abaissé chez le prématuré ou l'enfant de faible poids de naissance, et lorsqu'il existe ou a existé des conditions susceptibles d'altérer la barrière hémato-encéphalique immature, telle qu'une souffrance fœtale aiguë ou une acidose métabolique.

Dans la maladie hémolytique du nouveau-né, différents seuils sont proposés (grade $C$ ) :

- $\quad 340$ à $430 \mu$ mol. $L^{-1}$ de bilirubine totale chez le nouveau-né à terme ayant une immunisation $\mathrm{RH}$, à partir du troisième jour ;

- jusqu'à $540 \mu$ mol. $\mathrm{L}^{-1}$ dans les ictères observés chez le nouveau-né à terme sain, en l'absence d'immunisation RH.

Alternatives

La photothérapie représente le traitement de première intention des cas d'hyperbilirubinémie néonatale. Elle permet souvent d'éviter le recours à l'exsanguino-transfusion lorsqu'elle est intensive. Dans l'allo-immunisation fœto-maternelle, les transfusions in utero tendent à réduire les indications d'exsanguinotransfusion.

\section{Produits transfusés}

- Sang total ou reconstitué à partir de CGR conservés moins de 5 jours et de PFC. Un meilleur rendement transfusionnel n'a pas été prouvé avec des produits conservés moins de 3 jours.

Il est recommandé de recourir à des CGR :

- de groupe sanguin compatible avec ceux du nouveau-né et de sa mère ;

- phénotypés RH-KELL ;

- $\quad$ irradiés (nécessaires chez le prématuré et recommandés chez le nouveau-né à terme).

La déplasmatisation des CGR pourrait représenter une alternative à l'utilisation de CGR conservés moins de 7 jours, en ce qui concerne notamment les risques attribués à la solution de conservation.

Le recours à l'albumine humaine à la place du PFC pour la reconstitution du sang a été proposé. Mais, il n'existe pas d'étude établissant sa tolérance et son efficacité en matière de pouvoir tampon et de coagulation sanguine.

Le volume échangé est $\geq 2$ fois le volume sanguin total de l'enfant. La volémie du nouveau-né est habituellement de 80 $\mathrm{mL} \cdot \mathrm{kg}^{-1}$. 


\section{Alternatives à la transfusion homologue}

Les mesures d'épargne de transfusion de sang homologue comportent la transfusion autologue et l'érythropoïétine recombinante humaine (EPO). Le problème est d'en définir les indications appropriées. Aujourd'hui, on estime que les indications reposent sur la définition la plus précise possible des besoins transfusionnels individuels.

\subsection{Transfusion autologue}

\subsubsection{Transfusion autologue programmée (TAP)}

L'efficacité de la méthode à épargner la transfusion homologue est reconnue. Une TAP peut être envisagée chez toute personne de plus de $10 \mathrm{~kg}$ devant subir une intervention hémorragique programmée en l'absence des contre-indications (grade A). Les contre-indications sont les suivantes :

- celles exposant à un risque pour le malade :

- $\quad[\mathrm{Hb}]<11$ g.dL $\mathrm{dL}^{-1}$ ou $\mathrm{Ht}<33 \%$ (contre-indication à la mise en route ou à la poursuite du programme ;

- toute pathologie infectieuse latente ou patente (urinaire, digestive, dentaire, autre) ;

- malades porteurs chroniques de sonde urinaire ;

- pathologie cardiaque, notamment :

angor instable ;

crise angineuse dans les 8 jours précédents ;

rétrécissement aortique serré ;

cardiopathie cyanogène ;

- artériopathie cérébrale occlusive sévère.

- celles exposant à un risque collectif : sujets porteurs de marqueurs viraux directs, en raison du risque de contamination d'un autre patient en cas d'erreur d'attribution du produit ;

- refus du malade pour des raisons personnelles ou convictions religieuses.

Chez l'enfant : la TAP est difficile à réaliser en dessous de 15-20 kg en raison des problèmes d'abord veineux.

Chez la femme enceinte : ses indications sont rares et difficilement prévisibles. Les hémorragies du péripartum nécessitent souvent plusieurs CGR, rendant une transfusion homologue de complément le plus souvent nécessaire. L'indication est à envisager en cas de placenta acreta ou praevia recouvrant, de groupe sanguin rare, d'allo-immunisation et d'incompatibilité foeto-maternelle.

Au cours d'une intervention chirurgicale élective, une TAP peut être programmée avec un délai de 3 à 6 semaines, lorsque les pertes sanguines sont prévues excéder 1000 à $1500 \mathrm{~mL}$, comme par exemple : la chirurgie orthopédique (hanche, genou, chirurgie étendue du rachis), la chirurgie cardiaque, la chirurgie vasculaire majeure. La chirurgie carcinologique n'est pas une contre-indication.

En raison des multiples déterminants des pertes sanguines, il est indispensable que chaque établissement effectue une évaluation des besoins transfusionnels propres à chaque type de chirurgie pratiquée en routine, afin d'ajuster l'indication d'une TAP et le chiffre de CGR autologues nécessaires à sa pratique.

Les situations modifiant le rapport bénéfice/risque de la TAP doivent faire l'objet d'une concertation entre partenaires médicaux (anesthésiste-réanimateur, hémobiologiste, chirurgien) :

- le bénéfice escompté n'a généralement de sens qu'en cas d'espérance de vie $\geq 10$ ans ;

- des problèmes particuliers de transfusion homologue peuvent augmenter le rapport bénéfice/risque (disponibilité en sang homologue compatible dans un système de groupes sanguins rares, risque d'une allo-immunisation transfusionnelle).

La TAP n'élimine pas l'éventualité d'une transfusion homologue en cas de nécessité. Elle ne peut et ne doit être présentée au malade ni comme une garantie d'absence d'exposition à des PSL homologues, ni comme exempte de tous risques liés à la transfusion (notamment la contamination bactérienne).

Le prélèvement se fait par dons successifs à plusieurs semaines d'intervalle ou par un prélèvement unique par érythrocytaphérèse.

La supplémentation en fer doit être de règle en cas de TAP.

\subsubsection{Transfusion autologue par hémodilution normovolémique} intentionnelle

Par rapport à une hémodilution simple passive, l'économie de sang homologue que cette technique peut laisser espérer est, au mieux, modeste au prix d'un abaissement important de l'hémoglobinémie. La validation de son efficacité est insuffisante, à l'exception de la chirurgie cardiaque. Elle ne peut donc être considérée comme une alternative à la TAP.

Son utilisation en complément d'autres techniques peut être envisagée en cas de chirurgie très hémorragique. Elle n'est pas indiquée dans des chirurgies à saignement modéré $(<50 \%$ du volume sanguin), sauf cas particuliers (groupes sanguins rares, allo-immunisation transfusionnelle). Elle demande vigilance, expérience et connaissance des perturbations physiologiques et des risques inhérents à l'hémodilution profonde.

Elle est contre-indiquée chez les sujets ayant une tolérance réduite à l'abaissement de l'hémoglobinémie. La surveillance continue de la $\mathrm{Sv}_{2}$ est recommandée pour les hémodilutions profondes.

6.1.3 Transfusion autologue par récupération péri-opératoire Par récupération per-opératoire 
Cette technique paraît d'autant plus justifiée que la chirurgie est hémorragique, que la réalisation d'un programme de TAP est impossible ou ne parvient pas à l'autosuffisance. Elle trouve ses meilleures indications en chirurgie cardiaque et vasculaire ( $A c$ cord professionnel). Son utilisation en cas de chirurgie néoplasique et en obstétrique est débattue.

Elle est contre-indiquée en cas de champ opératoire infecté et en cas d'utilisation de colles biologiques.

La retransfusion de quantités importantes de sang recueilli dans la plaie opératoire et non lavé risque d'entraîner des complications. Les volumes maximaux tolérables de sang non lavé sont impossibles à préciser car sans doute variables avec le type de chirurgie et de saignement. La technique apparaît sûre pour des volumes n'excédant pas $1000 \mathrm{~mL}$. La retransfusion de volumes supérieurs nécessite un lavage.

L'utilisation de systèmes simples de récupération sans lavage dans la réanimation préhospitalière des hémothorax traumatiques est une technique de sauvetage documentée.

\section{Par récupération post-opératoire}

La transfusion autologue post-opératoire peut être effectuée en chirurgie prothétique de genou avec garrot, notamment lorsqu'une TAP est impossible (Accord professionnel). Son efficacité dans la chirurgie prothétique de hanche n'est pas établie. Son utilité en chirurgie cardiaque mériterait d'être réévaluée. Son intérêt doit être apprécié en fonction des pertes post-opératoires habituellement observées dans chaque centre.

Cette technique est contre-indiquée en cas d'infection, locale ou générale, et en cas d'insuffisance rénale.

La période de recueil doit être limitée aux 6 premières heures post-opératoires.

\subsubsection{Utilisation du sang placentaire autologue en} néonatalogie

Clampage retardé du cordon chez le prématuré à la naissance

Le clampage retardé du cordon permet de récupérer du sang placentaire et d'augmenter ainsi de 30 à $50 \%$ la masse sanguine. Les conditions optimales de réalisation, notamment la durée du délai et la position de l'enfant, ainsi que la tolérance des modifications volémiques entraînées sont en cours d'étude. Cette méthode simple d'épargne transfusionnelle ne doit pas retarder la mise en œuvre des gestes de réanimation lorsque ceux-ci sont indiqués et nécessite dans tous les cas une surveillance étroite du nouveau-né durant le temps de transfusion placento-néonatale.

Transfusion à partir de sang placentaire autologue

Le recueil du sang placentaire autologue au cordon ombilical (en vue d'une éventuelle transfusion ultérieure) dans le but de réduire les risques transfusionnels chez les prématurés pendant le premier mois de vie, est en cours d'étude. La faisabilité de cette méthode est conditionnée par les possibilités de mobilisation du personnel nécessaire au moment de la naissance. Le risque de contamination septique actuellement constaté indique la nécessité d'études complémentaires visant à améliorer la qualité des prélèvements et de la conservation du sang placentaire.

\subsection{Erythropoïétine (en dehors de l'insuffisance rénale chronique)}

\subsubsection{Utilisation au cours des hémopathies malignes et}

en oncologie

L'érythropoïetine (EPO) peut corriger l'anémie des cancers et des hémopathies malignes, qu'ils soient traités ou non par chimiothérapie, avec disparition ou diminution significative des besoins transfusionnels érythrocytaires. Il est recommandé de l'utiliser lorsque le risque d'anémie sévère est élevé et la probabilité de réponse à l'EPO est forte (grade $A$ ).

Le risque de survenue d'une anémie sévère sous chimiothérapie est d'autant plus important que le patient est déjà anémique, lymphopénique et porteur d'une altération de l'état général. La réponse au traitement est d'autant plus probable que la concentration d'EPO sérique est basse, le patient est porteur d'un myélome ou qu'il reçoit un traitement par cisplatine.

Le délai minimal d'obtention d'un début de réponse est de l'ordre de 2 à 3 semaines; il est prolongé en cas de syndrome inflammatoire. L'altération de la qualité de vie liée à l'anémie étant précoce, contrairement à l'attitude transfusionnelle classique, laquelle est le plus souvent prudente et tardive, il est souhaitable d'instaurer un traitement par EPO, lorsqu'il est décidé, dès les premiers stades de l'anémie.

\subsubsection{Utilisation dans le cadre d'une TAP}

Dans le cadre de la TAP, les indications documentées de l'EPO sont celles du libellé d'AMM, en association au fer par voie orale, voire intraveineuse (grade $A$ ).

\subsubsection{Utilisation péri-opératoire hors TAP}

Chez le patient modérément anémique $(10<[\mathrm{Hb}]<13$ g. $\left.\mathrm{dL}^{-1}\right)$, l'EPO diminue la transfusion homologue lors d'interventions entraînant des pertes sanguines de l'ordre de $1000 \mathrm{~mL}$. Il est fondamental de lui associer du fer, par voie orale, voire intraveineuse. En Europe, l'AMM de l'EPO hors TAP est limitée à la seule chirurgie orthopédique. Il n'existe pas d'arguments scientifiques pour exclure des interventions programmées entraînant des pertes sanguines prévisibles similaires.

Le risque de thrombose artérielle a été évoqué chez des malades opérés d'un pontage aorto-coronaires. Le terrain vasculaire grave est une contre-indication au traitement par EPO lorsque celle-ci n'est pas associée à la TAP. 


\subsubsection{Utilisation en néonatalogie}

L'EPO permet de réduire la fréquence et le volume des transfusions du prématuré.

Elle ne permet cependant pas d'éviter les transfusions de certains grands prématurés au cours des premières semaines de vie, en raison de son délai d'action. Il est possible que son bénéfice soit réduit en présence d'indication transfusionnelle restrictive et en cas de recours à un donneur unique pour des transfusions répétées.

Elle est recommandée dans la prévention de l'anémie du prématuré (Accord professionnel), principalement si le poids de naissance est $<1000 \mathrm{~g}$ ou l'âge gestationnel $<34$ semaines, selon les modalités suivantes :

- une administration précoce (début entre le troisième et le huitième jour), car l'effet de l'EPO sur [Hb] nécessite au moins 15 jours pour se manifester ;

- une dose de l'ordre de $750 \mathrm{U} \cdot \mathrm{kg}^{-1}$ par semaine, répartie en trois administrations par voie sous-cutanée $\mathbf{;}^{2}$

- un apport entéral précoce de fer, débutant à $2 \mathrm{~mL} \cdot \mathrm{kg}^{-1} \cdot \mathrm{j}^{-1} \mathrm{de}$ fer élément, et progressant jusqu'à 6 à $8 \mathrm{~mL} \cdot \mathrm{kg}^{-1} \cdot \mathrm{j}^{-1}$ au bout de 15 jours de traitement par EPO si la tolérance digestive le permet.

Toutefois, de nouvelles études sont nécessaires afin d'évaluer la réduction du nombre de donneurs après traitement par EPO.

\subsubsection{Anémie du post-partum}

L'utilisation de l'EPO dans le traitement de l'anémie per et post-partum est débattue. Compte tenu des résultats contradictoires des études, son utilisation ne peut être recommandée.

\subsection{Fer}

Chaque fois qu'est programmée une chirurgie prévue hémorragique, il est conseillé de la faire précéder d'un traitement martial par voie orale pendant une période de plusieurs semaines, lorsque c'est possible. Le traitement martial par voie orale reste le traitement de référence et de première intention de l'anémie modérée du per et du post-partum.

La place et les modalités du traitement martial par perfusion intraveineuse en post-opératoire et dans le post-partum restent à définir. Des données préliminaires semblent encourageantes, mais il n'existe pas d'étude la comparant à la voie orale. Il est recommandé de réaliser ce type d'études. Un échec et/ou une mauvaise observance du traitement martial per os, une intolérance digestive majeure aux sels de fer par voie orale ou une situation de malabsorption du fer sont les seules utilisations reconnues des fers-sucroses intraveineux (Accord professionnel).

\subsection{Transporteurs d'oxygène}

Actuellement, aucun des transporteurs d'oxygène, solutions d'hémoglobine ou fluorocarbones, n'est commercialisé. 
Annexe I. Principales caractéristiques des produits érythrocytaires et de leurs transformations

\begin{tabular}{|c|c|c|c|c|c|c|c|c|c|c|}
\hline \multirow[t]{3}{*}{ Produit } & \multirow[t]{3}{*}{ Type de transformation } & \multicolumn{3}{|c|}{$\begin{array}{l}\text { Contenu en hémoglobine } \\
\text { par produit }(\mathrm{g})\end{array}$} & \multicolumn{3}{|c|}{ Volume (mL) } & \multicolumn{3}{|c|}{ Durée de conservation (j) } \\
\hline & & \multirow[t]{2}{*}{$\min$} & \multirow[t]{2}{*}{ moy } & \multirow[t]{2}{*}{$\max$} & \multirow[t]{2}{*}{$\min$} & \multirow[t]{2}{*}{ moy } & \multirow[t]{2}{*}{$\max$} & \multicolumn{2}{|c|}{$\begin{array}{l}\text { système clos } \\
\text { avec adénine }\end{array}$} & \multirow[t]{2}{*}{ système ouvert } \\
\hline & & & & & & & & oui & non & \\
\hline $\begin{array}{l}\text { Sang total } \\
\text { déleucocyté }\end{array}$ & & $40^{\mathrm{a}}$ & & & 350 & & 563 & 7 & 7 & \\
\hline $\begin{array}{l}\text { CGR SAGM } \\
\text { déleucocyté UA }\end{array}$ & Déplasmatisé & $35^{d}$ & 51 & 66 & & & & & & $6 \mathrm{~h}$ \\
\hline $\begin{array}{l}\text { CGR SAGM } \\
\text { déleucocyté UA }\end{array}$ & Cryoconservé & 35 & 43 & 50 & & & & $7^{e}$ & & 1 \\
\hline $\begin{array}{l}\text { CGR SAGM } \\
\text { déleucocyté UA }\end{array}$ & Irradié & 40 & 54 & 70 & 230 & 280 & 410 & $42 / 1^{f}$ & & \\
\hline $\begin{array}{l}\text { CGR SAGM } \\
\text { déleucocyté UA }\end{array}$ & Sang reconstitué & 40 & 54 & 70 & 320 & & & & & 1 \\
\hline $\begin{array}{l}\text { CGR SAGM } \\
\text { déleucocyté UA }\end{array}$ & Préparation pédiatrique & & & & $50^{g}$ & & & 42 & & 1 \\
\hline $\begin{array}{l}\text { Sang total } \\
\text { déleucocyté UE }\end{array}$ & & $22^{\mathrm{a}}$ & & $<40$ & 283 & & 333 & 7 & 7 & \\
\hline $\begin{array}{l}\text { CGR } \\
\text { déleucocyté UE }\end{array}$ & & $22^{a}$ & & $<40$ & 85 & & 240 & 35 & 21 & \\
\hline $\begin{array}{l}\text { CGR SAGM } \\
\text { déleucocyté UE }\end{array}$ & & $22^{a}$ & & $<40$ & 155 & & 340 & 42 & & \\
\hline
\end{tabular}

a Valeurs réglementaires produits sanguins labiles (PSL) très rarement utilisé.

b Issu de sang total ou d'aphérèse.

c Données de la banque de données nationale de contrôle qualité des PSL.

d Données d'un établissement de l'Etablissement Français du Sang (EFS) [année 2001].

e Après décongélation et mise en solution de conservation.

f 42 jours après le prélèvement, si l'irradiation est faite avant le quinzième jour de conservation ; 24 heures après l'irradiation, si elle est faite à partir du quinzième jour de conservation.

g Chaque CGR transformé donne lieu à la préparation de plusieurs (minimum 4, maximum 8) préparations pédiatriques.

CGR = concentré de globules rouges $; \mathbf{h}=$ heure $; \mathbf{j}=$ jour $; \mathbf{m a x}=$ maximum $; \mathbf{m i n}=$ minimum $;$ moy = moyen $; \mathbf{S A G M}=$ milieu de conservation saline adénine, glucose, mannitol ; UA = unité adulte ; UE = unité enfant. 
Annexe II. Nomenclature des antigènes de groupes sanguins

Pour l'appellation du phénotype, le symbole du système de groupe sanguin est suivi par deux points et les nombres représentant les spécificités sont séparés par des virgules. Un résultat positif n'est pas indiqué, un résultat négatif est indiqué en faisant précéder le nombre du signe moins. II n'y a pas d'espace entre chaque nombre. Par exemple:

- Le phénotype RHD+C+E-C+e+,Cw- est écrit $\mathrm{RH}: 1,2,-3,4,5,-8$

- Le phénotype KELL K-k+, Kp(a+b-) est écrit KEL : $-1,2,3,-4$

Pour l'usage écrit et verbal de désignation de l'antigène, le numéro du système de groupe sanguin et le zéro placés à gauche du numéro de l'antigène peuvent être supprimés et il n'y a pas d'espace entre le symbole du système et le numéro. Par exemple RH1, RH46, KEL3.

La désignation des spécificités d'un anticorps inclut le mot « anti » suivi d'un tiret, le symbole alphabétique du système et le numéro de l'antigène sans espace entre chaque signe. Par exemple anti-E est écrit anti-RH3.

Pour les symboles des gènes et haplotypes, les caractères sont en italiques. Lorsque l'italique ne peut pas être utilisé, le symbole du système de

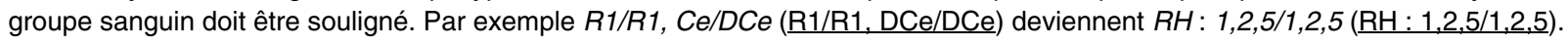

\begin{tabular}{|c|c|}
\hline \multirow{2}{*}{\multicolumn{2}{|c|}{$\begin{array}{l}\text { Correspondance des nomenclatu } \\
\text { Antigènes du système Rhésus }\end{array}$}} \\
\hline & \\
\hline $\mathrm{D}$ & $\mathrm{RH} 1$ \\
\hline $\mathrm{C}$ & $\mathrm{RH} 2$ \\
\hline$E$ & $\mathrm{RH} 3$ \\
\hline$c$ & $\mathrm{RH} 4$ \\
\hline e & $\mathrm{RH} 5$ \\
\hline \multicolumn{2}{|c|}{ Antigènes du système KELL } \\
\hline $\mathrm{K}$ & KEL1 \\
\hline $\mathrm{k}$ & KEL2 \\
\hline \multicolumn{2}{|c|}{ Antigènes du système Duffy } \\
\hline$F y^{a}$ & FY1 \\
\hline $\mathrm{Fy}^{\mathrm{b}}$ & FY2 \\
\hline \multicolumn{2}{|c|}{ Antigènes du système Kidd } \\
\hline $\mathrm{Jk}^{\mathrm{a}}$ & $\mathrm{JK} 1$ \\
\hline $\mathrm{Jk}^{\mathrm{b}}$ & JK2 \\
\hline \multicolumn{2}{|c|}{ Antigènes du système MNS } \\
\hline$M$ & MNS1 \\
\hline $\mathrm{N}$ & MNS2 \\
\hline$S$ & MNS3 \\
\hline s & MNS4 \\
\hline \multicolumn{2}{|c|}{ Antigènes du système $P$} \\
\hline $\mathrm{P} 1$ & P1 \\
\hline \multicolumn{2}{|c|}{ Antigènes du système Lewis } \\
\hline $\operatorname{Le}^{\mathrm{a}}$ & LE1 \\
\hline $\mathrm{Le}^{\mathrm{b}}$ & LE2 \\
\hline
\end{tabular}


Annexe III. Organisation générale de la transfusion en urgence ${ }^{3}$

II convient que les établissements de santé et les sites transfusionnels précisent les modalités à mettre en place pour répondre au mieux à l'urgence. II revient :

\section{Aux établissements de santé de s'organiser pour :}

Disposer des résultats au moment opportun.

Avertir le site transfusionnel du caractère de l'urgence : urgence vitale immédiate, urgence vitale ou urgence "relative ", afin que toutes les procédures soient mises en œuvre immédiatement.

Avertir le site transfusionnel en cas de recherche d'agglutinines irrégulières (RAI) positive, afin que des produits sanguins compatibles soient préparés le plus rapidement possible.

Dépister les usurpations d'identité et signaler les corrections d'orthographe.

\section{Aux sites transfusionnels de s'organiser pour :}

Reconnaître les prescriptions de produits sanguins labiles (PSL) relevant d'une urgence vitale immédiate, d'une urgence vitale ou d'une urgence " relative » et y répondre par une distribution adéquate : immédiate, différée ou acheminement de précaution vers le dépôt.

Mettre dès réception des échantillons les examens immuno-hématologiques en œuvre, en communiquant les résultats biologiques au prescripteur le plus rapidement possible.

Prendre en compte un résultat de RAI négatif réalisé dans un autre laboratoire que celui du site distributeur (si l'analyse a été réalisée selon des critères réglementaires).

A la fois à l'établissement de santé et au site transfusionnel :

Analyser dans chaque région le maillage entre les services d'urgence et les sites transfusionnels afin de pouvoir disposer de concentré de globules rouges (CGR) dans un délai inférieur ou égal à 30 minutes, « tout au long de l'année et 24 heures sur 24 ». Pour l'obtention de CGR dans le cadre de l'urgence vitale ou de l'urgence vitale immédiate (en moins de 30 minutes), trois solutions sont possibles : un site transfusionnel de l'EFS est situé à proximité, un dépôt de sang ayant une autorisation d'attribution est situé dans l'établissement de santé ou un dépôt d'urgence vitale autorisé existe dans l'établissement sous des conditions strictement réglementées.

Etablir la quantité et la qualité des stocks d'urgence vitale. Le stock doit être volontairement réduit (son but est d'assurer la survie du patient pendant le temps d'acheminement des autres CGR) ; il est conseillé qu'il ne soit constitué que de 2 CGR O RH : $-1,-2,-3 \mathrm{KEL}:-1$ (anciennement ccddee, K-) et de 2 CGR O RH : 1,2,-3,-4,5 KEL : -1 (anciennement CCDee, K-).

Réaliser avec l'ensemble des acteurs concernés des procédures de prescription d'urgence vitale tant au niveau de l'établissement de soins que de sa réponse au niveau du site transfusionnel, et s'assurer de leur bon fonctionnement. Il est recommandé de clarifier, entre autres, l'ensemble des modalités de communication entre les établissements de santé et les sites transfusionnels.

Prévoir le réapprovisionnement du dépôt d'urgence.

Organiser les transports conformément aux bonnes pratiques de transport des prélèvements, produits et échantillons issus du sang humain en précisant (obligation réglementaire) : - qui apporte les examens, - qui transporte le sang, - dans quels délais (définition du délai minimum d'obtention des produits sanguins en incluant le temps de transport et les délais incompressibles de distribution en fonction des examens nécessaires), - les horaires d'ouverture, - les moyens de transport (type de véhicule et de containers, les modes de contrôle des températures...), - qui réapprovisionne le dépôt et selon quelles modalités. Il conviendra d'établir un cahier des charges précis et un contrat d'engagement devra définir clairement les différents points du transport liant : l'établissement de santé, le site transfusionnel et le transporteur.

L'évaluation de l'efficacité des mesures mises en place doit être clairement définie dans un calendrier : hebdomadaire dans un premier temps, mensuelle, puis trimestrielle et sera réalisée par l'établissement de santé et par le site transfusionnel.

Il est recommandé que toute trace écrite des éventuels événements figure dans le dossier clinique, à des fins d'enquête ou d'évaluation ultérieure.

3 D'après les conclusions de la table ronde organisée par l'Etablissement Français du Sang (EFS) sur le traitement des urgences transfusionnelles obstétricales. 


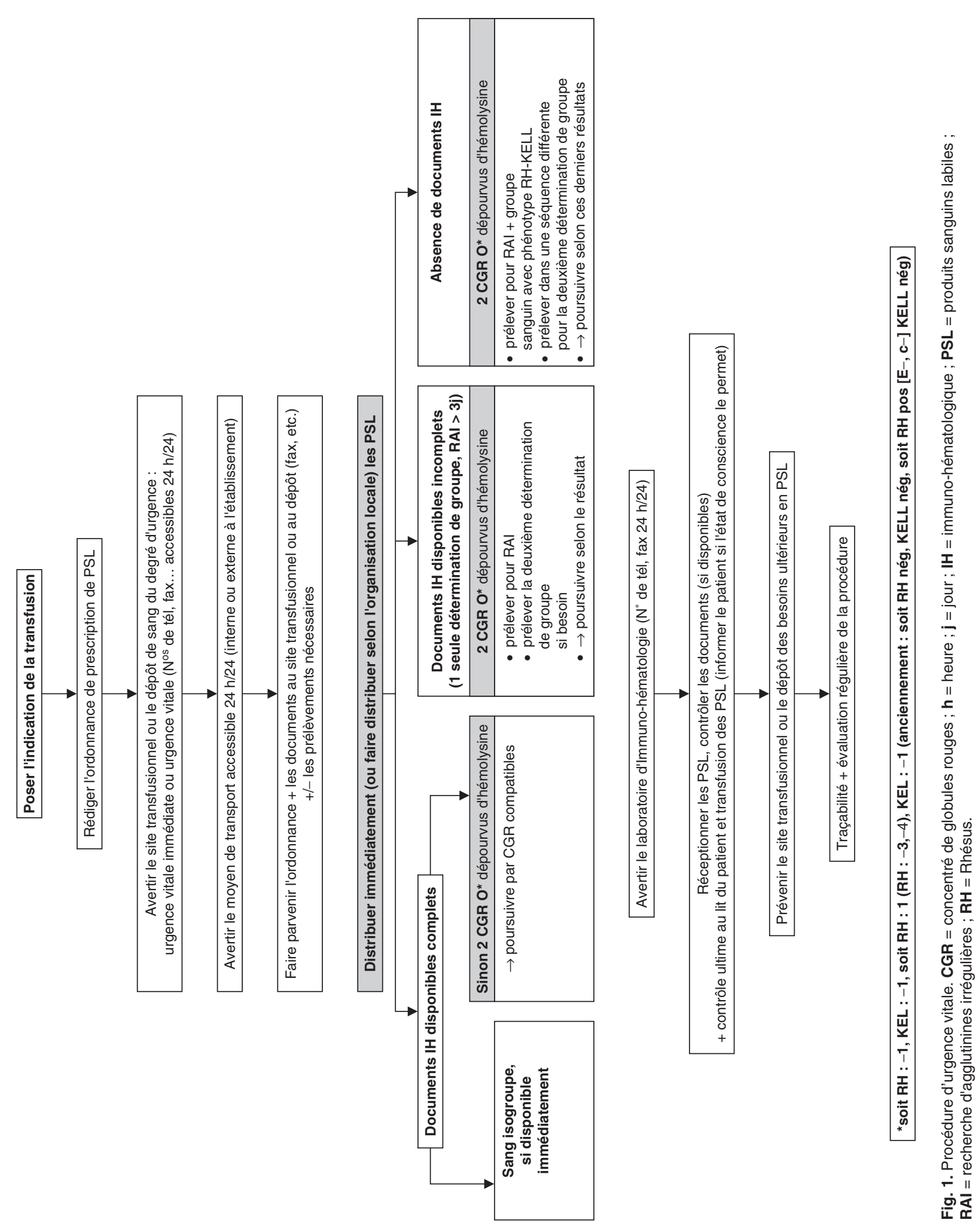

